\title{
Operator Product EXPANSION IN Logarithmic Conformal Field Theory
}

\author{
Micћael Flohr \\ Institute for Theoretical Physics, University of Hannover \\ Appelstraße 2, D-30167 Hannover, Germany \\ E-mail: flohr@itp.uni-hannover.de
}

March 20, 2002

\begin{abstract}
In logarithmic conformal field theory, primary fields come together with logarithmic partner fields on which the stress-energy tensor acts non-diagonally. Exploiting this fact and global conformal invariance of two- and three-point functions, operator product expansions of logarithmic operators in arbitrary rank logarithmic conformal field theory are investigated. Since the precise relationship between logarithmic operators and their primary partners is not yet sufficiently understood in all cases, the derivation of operator product expansion formulæ is only possible under certain assumptions. The easiest cases are studied in this paper: firstly, where operator product expansions of two primaries only contain primary fields, secondly, where the primary fields are pre-logarithmic operators. Some comments on generalization towards more relaxed assumptions are made, in particular towards the case where logarithmic fields are not quasi-primary. We identify an algebraic structure generated by the zero modes of the fields, which proves useful in determining settings in which our approach can be successfully applied.
\end{abstract}

* Research supported by EU TMR network no. FMRX-CT96-0012 and the DFG String network (SPP no. 1096), Fl 259/2-1. 


\section{INTRODUCTION}

DURING THE LAST few years, so-called logarithmic conformal field theory (LCFT) established itself as a well-defined new animal in the zoo of conformal field theories in two dimensions. To our knowledge, logarithmic singularities in correlation functions were first noted by Knizhnik back in 1987 41. Although many features such as logarithmic divergences of correlators and indecomposable representations were observed in various places, most notably in 74, 73, it took six years, until the concept of a conformal field theory with logarithmic divergent behavior was introduced by Gurarie [27. From then one, there has been a considerable amount of work on analyzing the general structure of LCFTs, which by now has generalized almost all of the basic notions and tools of (rational) conformal field theories, such as null vectors, characters, partition functions, fusion rules, modular invariance etc., to the logarithmic case, see for example 16, 34, 21, 67, 72, 23, 46, 68, 13, 38, 58, 60, 25] and references therein. Besides the best understood main example of the $\operatorname{logarithmic} c=-2$ theory and its $c_{p, 1}$ relatives, other specific models were considered such as WZW models [1, 45, 64, 65, 22] and LCFTs related to supergroups and supersymmetry [73, 11, 39, 37, 55, 2, 71, 51].

Also, quite a number of applications have already been pursued, and LCFTs have emerged in many different areas by now. Sometimes, longstanding puzzles in the description of certain theoretical models could be resolved, e.g. the Haldane-Rezayi state in the fractional quantum Hall effect [28, 70, 70], multi-fractality [12], or two-dimensional conformal turbulence [18, 66, 76]. Other applications worth mentioning are gravitational dressing [5], polymers and abelian sandpiles [74, 33, 8, 57], the (fractional) quantum Hall effect [17, 31, 49], and - perhaps most importantly - disorder [9, 43, 56, 29, 19, 69, 30, 3, 4]. Finally, there are even applications in string theory [42], especially in $D$-brane recoil 14, 44, 15, 59, 52, 6, 53, 26], AdS/CFT correspondence [24, 40, 35, 47, 63, 48, 75, 62], as well as in Seiberg-Witten solutions to supersymmetric Yang-Mills theories, e.g. [19],

Last, but not least, a recent focus of research on LCFTs is in its boundary conformal field theory aspects [60, 50, 54, 32, 36].

However, the computation of correlation functions within an LCFT still remains difficult, and only in a few cases, four-point functions (or even higher-point functions) could be obtained explicitly. The main reason for this obstruction is that the representation theory of the Virasoro algebra is much more complicated in the LCFT case due to the fact that there exist indecomposable but non-irreducible representations (Jordan cells). This fact has many wide ranging implications. First of all, it is responsible for the appearance of logarithmic singularities in correlation functions. Furthermore, it makes it necessary to generalize almost every notion of (rational) conformal field theory, e.g. characters, highestweight modules, null vectors etc. We note here that indecomposable representations need not occur with respect to the Virasoro algebra, but may occur with respect to (part of) a extended chiral symmetry algebra such as current algebras or $\mathcal{W}$-algebras. For the sake of simplicity, we will confine ourselves in this paper to the case where Jordan cells are with respect to the Virasoro algebra.

In particular, what was lacking so far is a consistent generic form of operator product expansions (OPEs) between arbitrary rank logarithmic fields. Although such OPEs can be derived from co-product considerations in the purely representation theoretical framework [34, 21], a direct approach trying to fix the generic form from global conformal covariance of the fields is clearly desirable. For the simple case of a rank two LCFT, where Jordan cells are two-dimensional, it was known since some time 27, 9] that the two-point functions 
of a primary $\Psi_{(h ; 0)}(z)$ and its only logarithmic partner $\Psi_{(h ; 1)}(z)$ are

$$
\begin{aligned}
& \left\langle\Psi_{(h ; 0)}(z) \Psi_{(h ; 0)}(w)\right\rangle=0, \\
& \left\langle\Psi_{(h ; 0)}(z) \Psi_{(h ; 1)}(w)\right\rangle=D_{(h, h ; 1)}(z-w)^{-2 h}, \\
& \left\langle\Psi_{(h ; 0)}(z) \Psi_{(h ; 1)}(w)\right\rangle=\left[D_{(h, h ; 2)}-2 D_{(h, h ; 1)} \log (z-w)\right](z-w)^{-2 h} .
\end{aligned}
$$

However, as we shall see, even in this simple case OPEs turn out to be more complicated, and one needs all possible three-point functions as well. First results in this direction can be found in [67, 23, 38, 61]. Here we will start to close this gap and provide the general structure of OPEs for fields constituting arbitrary rank Jordan cells under certain assumptions.

Let us briefly outline the basic problem: In ordinary conformal field theory, the generic structure of the operator product expansion is fixed up to structure constants which depend only on the conformal weights of the fields involved,

$$
\Phi_{h_{i}}(z) \Phi_{h_{j}}(w)=\sum_{k} C_{i j}^{k}(z-w)^{h_{k}}\left(\Phi_{h_{k}}(w)+\sum_{\{n\}} \beta_{i j}^{k,\{n\}}(z-w)^{|\{n\}|} \Phi_{h_{k}}^{(-\{n\})}(w)\right) .
$$

Here, the fields $\Phi_{h}$ are primaries, and the coefficients $\beta_{i j}^{k,\{n\}}$ of the descendant contributions $\Psi_{h}^{(-\{n\})}=L_{-\{n\}} \Phi_{h}=L_{-n_{1}} L_{-n_{2}} \ldots L_{-n_{l}} \Phi_{h}$ are entirely fixed by conformal covariance. The point is that the structure constants $C_{i j}^{k}$ can be easily determined if the two- and three-point functions are known. In fact, these define constants $D_{i j}=$ $\left\langle\Psi_{h_{i}}(\infty) \Psi_{h_{j}}(0)\right\rangle$ and $C_{i j k}=\left\langle\Psi_{h_{i}}(\infty) \Psi_{h_{j}}(1) \Psi_{h_{k}}(0)\right\rangle$ respectively, where $D_{i j}$ is usually a diagonal matrix, i.e. $D_{i j} \propto \delta_{h_{i}, h_{j}}$. The two-point functions define a metric on the space of fields, such that this metric $D_{i j}$ and its inverse can be used to lower and raise indices (in field space) respectively. In particular, the OPE structure constants are simply given by

$$
C_{i j}^{k}=C_{i j l} D^{l k}
$$

Now, in logarithmic conformal field theory, the metric induced by the two-point functions is no longer diagonal - see (1.1) for the simplest case, where the metric, restricted to fixed conformal weight $h$, is of the form $\left(\begin{array}{cc}0 & D_{1} \\ D_{1} & D_{2}-2 D_{1} \log (\cdot)\end{array}\right)(\cdot)^{-2 h}$. Note that, even worse, the metric cannot any longer be factorized in a coordinate dependent part and a purely constant part. It is the purpose of this paper to work out this metric together with all needed three-point functions in order to find the correct equivalent to (1.3) in the logarithmic case.

The paper will proceed as follows: In the next section, we will set up our notation and outline the different cases we will study in the following, progressing from the simplest setting to some more involved indecomposable structures. Although we restrict ourselves to indecomposable representations with respect to the Virasoro algebra alone, we will encounter a surprisingly rich set of possibilities of which only the easier ones can be treated with the methods presented in this paper.

In the third section, we start the investigation in the simplest possible setting, i.e. where all logarithmic partner fields in a Jordan cell are assumed to be quasi-primary. This setting is as close as possible to the ordinary CFT case, and the generic form of one-, two-, and three-point functions can be computed explicitly, as long as the primary fields within the Jordan cells are assumed to be proper primaries. We also comment on the 
appropriate definition of a Shapovalov form, and show that it is well defined under the above assumptions on the structure of two-point functions.

In section four we derive the generic structure of OPEs in this setting. Although we usually work only with the holomorphic part of the LCFT, we briefly discuss locality in this section. We also compute a fully elaborated example of a rank four LCFT with only proper primary fields involved, demonstrating some perhaps unexpected features of operator products in the logarithmic case.

Then, in the fifth section, we consider two more general cases, where the primary fields are not proper primaries, meaning that an OPE between two primary fields may contain a logarithmic field. Both examples, fermionic fields and twist fields, already occur in the best known LCFT, namely the $c=-2$ system, which will serve as our prime example. We show that OPEs can be computed for these cases as well, since these primary fields are not part of Jordan cells.

Finally, section six is devoted to the question, how the so far quite restrictive assumptions can be relaxed. In particular, we investigate, under which circumstances logarithmic fields can be non-quasi-primary without affecting the results of the preceding sections. We also briefly address the other assumptions we made and discuss the difficulties one would have to face attempting to derive the generic structure of OPEs and correlation functions without them. In particular, we demonstrate that the case of Jordan cells containing primary fields which are not proper primaries cannot be solved without further assumptions on the structure of the CFT. Thus, we conclude that global conformal covariance fixes the generic form of correlation functions and OPEs in a similar fashion as in the ordinary CFT case only when the indecomposable representations have a rather simple form.

We conclude with a brief discussion of our results and possible directions for future research.

\section{DEFINITIONS AND PRELIMINARIES}

TO START WITH, we fix some notation. In general, a rank $r$ Jordan cell with respect to the Virasoro algebra is spanned by $r$ states $\{|h ; r-1\rangle, \ldots,|h ; 1\rangle,|h ; 0\rangle\}$ with the property

$$
L_{0}|h ; k\rangle=h|h ; k\rangle+\left(1-\delta_{k, 0}\right)|h ; k-1\rangle .
$$

These states are defined via $\lim _{z \rightarrow 0} \Psi_{(h ; k)}(z)|0\rangle=|h ; k\rangle$, where $|0\rangle$ denotes the $\operatorname{SL}(2, \mathbb{C})$ invariant vacuum with $L_{n}|0\rangle=0 \forall n \geq-1$. The fields $\Psi_{(h ; k)}(z)$ with $0<k<r$ are the so-called logarithmic partner fields. The field $\Psi_{(h ; 0)}(z)$ is a primary field which forms the only proper irreducible sub-representation within the module of descendants of the Jordan cell. In the following we will denote the primary by $\Phi_{h}(z) \equiv \Psi_{(h ; 0)}(z)$, if and only if it is a proper primary field. We call a primary field proper primary, if its OPE with other proper primary fields never produces a logarithmic field on the right hand side. This definition is motivated from examples of LCFTs, where the primary fields within Jordan cells do share precisely this property. For instance, in the prime LCFT example, the $c=-2$ theory, there exists a Jordan cell of rank two for $h=0$. The primary field is the identity field which by definition is a proper primary.

For completeness, we note that within a logarithmic CFT, Jordan cells of different rank might occur, i.e. $r=r(h)$ might be a function of the conformal weight of the corresponding (proper) primary field. Of course, if $r=1$, the Jordan cell reduces to an ordinary highest weight state, and its module of descendants to an ordinary Verma module. For more 
precise definitions see [72]. However, we will see later that consistency of the operator algebra makes it virtually impossible that Jordan cells of different rank occur within the same LCFT.

We have to distinguish between the proper primary fields $\Phi_{h}(z) \equiv \Psi_{(h ; 0)}(z)$ in a Jordan cell and so-called pre-logarithmic primary fields. Pre-logarithmic fields are Virasoro primary fields, whose operator product expansions among themselves might lead to logarithmic fields [46]. Typically, pre-logarithmic fields turn out to be twist fields. Although no counter example is known, we cannot exclude that pre-logarithmic fields might occur as primaries within a non-trivial Jordan cell. However, for the purposes of this paper, we will not assume so for the beginning. Thus, initially we will assume that primary fields in non-trivial Jordan cells are all proper primaries. Later, in section five, we will discuss the case of pre-logarithmic fields.

We also say that the field $\Psi_{(h ; k)}$ has Jordan level $k$ in its Jordan cell, abbreviated as J-level $k$. Proper primary fields have J-level zero by definition. Twist fields do not possess a well-defined J-level. Instead, they carry a fractional charge $q=\ell / n$ whose denominator denotes the branching number. Logarithmic operators can appear in OPEs of twist fields $\chi_{h(q)}$ and $\chi_{h\left(q^{\prime}\right)}$, whenever $q+q^{\prime} \in \mathbb{Z}$.

As discussed by F. Rohsiepe [72], the possible structures of indecomposable representations with respect to the Virasoro algebra are surprisingly rich. Besides the defining condition (2.1), further conditions have to be employed to fix the structure. The simplest case is defined via the additional requirement

$$
L_{1}|h ; k\rangle=0, \quad 0 \leq k<r .
$$

This condition means that all fields spanning the Jordan cell are quasi-primary. It will be our starting point in the following.

The next complicated case is where logarithmic partners are not necessarily quasiprimary. An example is again provided by the simplest explicitly known LCFT, the $c=-2$ model. It possesses a rank two Jordan cell at $h=1$ where the logarithmic partner is not quasi-primary, i.e. $L_{0}|h=1 ; 1\rangle=|h=1 ; 1\rangle+|h=1 ; 0\rangle$ and $L_{1}|h=1 ; 1\rangle=|\xi\rangle \neq 0$, see 21] for details. In general, this spoils attempts to make use of the global conformal Ward identities, since these are consequences of global conformal covariance or, in other words, of quasi-primarity of the fields. However, it seems that the condition of quasiprimary fields can be relaxed under certain circumstances: If the state $|\xi\rangle$ generated by the action of $L_{1}$ on $|h=1 ; 1\rangle$ does not have a non-vanishing product with any of the states involved so far, it will not affect the form of the conformal Ward identities. In the given example, this is the case. We will postpone a more detailed discussion of this to the last section, but will give some definitions motivated by an example, which will prove useful for this later discussion.

\section{II.1. An Example And Zero Mode Content}

In all known examples of LCFTs, the occurrence of indecomposable representations can be traced back to certain pairs of conjugate zero modes. Let us explain this within the example of the LCFT with central charge $c=c_{2,1}=-2$. It may be described, see for instance [28], by two anti-commuting spin zero fields or ghost fields $\theta_{\alpha}, \alpha= \pm$, with the $S L(2, \mathbb{C})$ invariant action

$$
S \propto \mathrm{i} \int \mathrm{d}^{2} z \varepsilon^{\alpha \beta} \partial \theta_{\alpha} \bar{\partial} \theta_{\beta}
$$


In order to quantize this theory, one has to compute the fermionic functional integral

$$
\mathcal{Z}=\int \mathcal{D} \theta_{0} \mathcal{D} \theta_{1} \exp (-S)
$$

This fermionic path integral, when computed formally, vanishes due to the zero modes of the $\theta$ fields, which do not enter the action. To make it non-zero, the zero-modes have to be added by inserting the $\theta$ fields into correlation functions,

$$
\mathcal{Z}^{\prime}=\frac{1}{2} \int \mathcal{D} \theta_{0} \mathcal{D} \theta_{1} \varepsilon^{\alpha \beta} \theta_{\alpha} \theta_{\beta} \exp (-S)=1
$$

As an immediate consequence, the vacuum of this theory behaves in an unusual way, since its norm vanishes, $\langle 0 \mid 0\rangle=0$, while the explicit insertion of the $\theta$ fields produces a non-zero result, $\frac{1}{2}\left\langle\varepsilon^{\alpha \beta} \theta_{\alpha}(z) \theta_{\beta}(w)\right\rangle=1$. More generally, these insertions are also necessary when arbitrary correlation functions involving only derivatives $\partial \theta$ are computed, since derivatives cancel the zero modes, i.e. the constant parts of the $\theta$ fields. Therefore, we have

$$
\frac{1}{2}\left\langle\varepsilon^{\alpha \beta} \partial \theta_{\alpha}(z) \partial \theta_{\beta}(w)\right\rangle=0, \quad \text { but } \quad \frac{1}{4}\left\langle\varepsilon^{\alpha \beta} \partial \theta_{\alpha}(z) \partial \theta_{\beta}(w) \varepsilon^{\gamma \delta} \theta_{\gamma}(0) \theta_{\delta}(0)\right\rangle=\frac{-1}{(z-w)^{2}},
$$

where the latter correlator is computed in analogy to the free bosonic field. From the viewpoint of conformal field theory, this strange behavior can be explained in terms of logarithmic operators which naturally appear in the $c=-2$ theory. As shown in [27], this CFT must necessarily possess an operator $\tilde{\mathbb{I}}$ of scaling dimension zero in addition to the unit operator $\mathbb{I}$, such that $\left[L_{0}, \tilde{\mathbb{I}}\right]=\mathbb{I}$ with $L_{0}$ (half of) the Hamiltonian. This property alone necessarily leads to the correlation functions

$$
\langle\mathbb{I} \mathbb{I}\rangle=0, \quad\langle\mathbb{I}(z) \tilde{\mathbb{I}}(w)\rangle=1, \quad\langle\tilde{\mathbb{I}}(z) \tilde{\mathbb{I}}(w)\rangle=-2 \log (z-w),
$$

which can be proved by general arguments of conformal field theory such as global conformal invariance and the operator product expansion. Furthermore, it follows that the field $\tilde{\mathbb{I}}$ can indeed be identified with the normal ordered product of the $\theta$ fields, i.e.

$$
\tilde{\mathbb{I}}(z)=-\frac{1}{2} \varepsilon^{\alpha \beta}: \theta_{\alpha} \theta_{\beta}:(z) .
$$

The stress energy tensor of this CFT is given by the normal ordered product

$$
T(z)=\frac{1}{2} \varepsilon^{\alpha \beta}: \partial \theta_{\alpha} \partial \theta_{\beta}:(z),
$$

and is easily seen to fulfill the correct operator product expansion with itself yielding the central charge $c=-2$. To fix notation, the mode expansions of the $\theta$ fields read

$$
\theta_{\alpha}(z)=\xi_{\alpha}+\theta_{\alpha, 0} \log (z)+\sum_{n \neq 0} \theta_{\alpha, n} z^{-n}
$$

where the $\xi$ 's are the crucial zero modes. The above mode expansion is valid in the untwisted sector (periodic boundary conditions), where $n \in \mathbb{Z}$. In the twisted sector (anti-periodic boundary conditions) $n \in \mathbb{Z}+\frac{1}{2}$, and no zero modes are present. The anti-commutation relations read for the case $\alpha \neq \beta$ in both sectors

$$
\begin{aligned}
\left\{\theta_{\alpha, n}, \theta_{\beta, m}\right\} & =\frac{1}{n} \delta_{n+m, 0} \text { for } n \neq 0, \\
\left\{\theta_{\alpha, 0}, \theta_{\beta, 0}\right\} & =\left\{\xi_{\alpha}, \xi_{\beta}\right\}=0, \\
\left\{\xi_{\alpha}, \theta_{\beta, 0}\right\} & =1
\end{aligned}
$$


with all other anti-commutators vanishing. Note that the $\xi$-modes become the creation operators for logarithmic states. Indeed, the highest weight conditions of the standard $S L(2, \mathbb{C})$ invariant vacuum are

$$
\theta_{\alpha, n}|0\rangle=0 \quad \forall n \geq 0
$$

such that

$$
|\tilde{0}\rangle=\tilde{\mathbb{I}}(0)|0\rangle=-\frac{1}{2} \varepsilon^{\alpha \beta} \xi_{\alpha} \xi_{\beta}|0\rangle .
$$

It is instructive to conclude our example with the little exercise to compute $L_{0}|\tilde{0}\rangle$ by using the mode expansion $L_{n}=\frac{1}{2} \sum_{m} \varepsilon^{\alpha \beta}: \theta_{\alpha, n-m} \theta_{\beta, m}$ : as follows:

$$
\begin{aligned}
L_{0}|\tilde{0}\rangle & =-\frac{1}{4} \varepsilon^{\alpha \beta} \varepsilon^{\gamma \delta} \sum_{m}:\left(\delta_{m, 0}-m^{2}\right) \theta_{\alpha,-m} \theta_{\beta, m}: \xi_{\gamma} \xi_{\delta}|0\rangle \\
& =+\frac{1}{4} \varepsilon^{\alpha \beta} \varepsilon^{\gamma \delta}\left(\xi_{\gamma} \xi_{\delta} \sum_{m>0} m^{2}\left(\theta_{\alpha,-m} \theta_{\beta, m}-\theta_{\beta,-m} \theta_{\alpha, m}\right)-\theta_{\alpha, 0} \theta_{\beta, 0} \xi_{\gamma} \xi_{\delta}\right)|0\rangle \\
& =-\frac{1}{4} \varepsilon^{\alpha \beta} \varepsilon^{\gamma \delta} \theta_{\alpha, 0} \theta_{\beta, 0} \xi_{\gamma} \xi_{\delta}|0\rangle=\frac{1}{2} \varepsilon^{\alpha \beta} \varepsilon_{\alpha \beta}|0\rangle=|0\rangle,
\end{aligned}
$$

where the third equality follows from the highest weight condition (2.12), and otherwise the anti-commutation relations (2.11) were used. This clearly demonstrates that the states $|\tilde{0}\rangle$ and $|0\rangle$ form an indecomposable Jordan cell with respect to the Virasoro algebra. The action of other Virasoro modes can be computed in the same fashion.

What we learn from this example is the crucial role of conjugate pairs of zero modes, i.e. pairs $\xi_{\alpha}, \theta_{\beta, 0}$. It turns out that logarithmic fields are precisely those fields, whose mode expansion contains $\varepsilon^{\alpha \beta} \xi_{\alpha} \xi_{\beta}$. Therefore, it makes sense to talk of the "logarithmicity" of a field, more precisely of its $\xi$ zero mode content. It is clear that there might be more pairs of conjugate modes in more general LCFTs (e.g. in higher spin ghost system) and hence a field might possess a higher zero mode content. The phrase zero mode is ambiguous: We do not mean the zero-th mode in the mode expansion of a field, but pairs of conjugate modes $a_{n}, c_{-n}$ such that $a_{n}$ annihilates to both sides, i.e. $\left\langle 0\left|a_{n}=a_{n}\right| 0\right\rangle=0$, and $c_{-n}$ is a creator to both sides. In the example, $\theta_{\alpha, 0}$ are the annihilators and $\xi_{\beta}$ the creators. The zero mode content of a field counts the number of creation operator zero modes. If the modes are anti-commuting, we will also talk of an even or odd zero mode content of fields. Fields of even zero mode content are called bosonic, fields of odd zero mode content fermionic, respectively. It is important to note that a correlation function can only be non-vanishing, if its total zero mode content is large enough to kill all annihilator zero modes. In our example, any non-vanishing correlator must contain $\varepsilon^{\alpha \beta} \xi_{\alpha} \xi_{\beta}$. Moreover, our example is of fermionic nature, such that the zero mode content must always be even.

To fix notation, we will denote the number of creator zero modes of a field $\Psi$ by $Z_{0}(\Psi)$. If the modes are fermionic, we further introduce $Z_{-}(\Psi)$ and $Z_{+}(\Psi)$ which count the modes with respect to the spin doublet label $\alpha= \pm$ respectively. Of course, $Z_{0}(\Psi)=$ $Z_{-}(\Psi)+Z_{+}(\Psi)$ in this case. We stress that correlation functions $\left\langle\Psi_{1}\left(z_{1}\right) \ldots \Psi_{n}\left(z_{n}\right)\right\rangle$ of fields $\Psi_{i}\left(z_{i}\right)$ with $Z_{0}\left(\Psi_{i}\right)=0$ for all $i=1, \ldots, n$ must vanish. Moreover, if the minimal zero mode content of a given LCFT is $N$, all correlators vanish, whenever $\sum_{i} Z_{0}\left(\Psi_{i}\right)<N$. Thus, such fields behave almost as null fields. They are not entirely null, since we may insert those fields in a correlator with already sufficiently high total zero mode content without necessarily forcing it to become zero. In our example, $N=N_{-}+N_{+}=1+1=2$. The rank of Jordan cells of a LCFT generated by anti-commuting fields is $N / 2+1$, otherwise it should be $N+1$, as in the case of LCFTs from puncture operators in Liouville theories [14, 43, 46]. 


\section{II.2. Zero Mode Content And J-Level}

We will discuss in section six that the zero mode content allows to put bounds on the Jlevels of fields appearing on the right hand side of OPEs. However, it is not clear whether all LCFTs admit to assign a zero mode content in the above defined sense to all of its fields. Thus, the basic problem one is faced with is that there is no a priori rule that restricts the J-level in an OPE of the form

$$
\Psi_{\left(h_{1} ; k_{1}\right)}(z) \Psi_{\left(h_{2} ; k_{2}\right)}(w)=\sum_{h} \sum_{k=0}^{r(h)-1} C_{\left(h_{1} ; k_{1}\right)\left(h_{2} ; k_{2}\right)}^{(h ; k)}(z-w)^{h-h_{1}-h_{2}} f_{k_{1} k_{2}}^{k}(z-w) \Psi_{(h ; k)}(w),
$$

where the functions $f_{k_{1} k_{2}}^{k}(x)$ collect possible logarithmic terms. What one would definitely wish for would be something like a gradation such that the J-level of the right hand side of (2.15) is bounded as

$$
k \leq k_{1}+k_{2} .
$$

Unfortunately, we know that this is not always true. Pre-logarithmic fields, for instance, are true primary fields which produce a logarithmic field in their OPE which means that $1=k>k_{1}+k_{2}=0+0$ in contradiction to the above bound.

Now, if the LCFT under consideration admits to assign a well-defined zero mode content to each of its fields, we can trace back the origin of logarithms and of Jordan cells to precisely that zero mode content, as indicated in the preceding subsection. Since the Jordan cell structure is in this case generated by the existence of certain creator zero modes, it is clear that the OPE of two fields can never produce fields on the right hand side whose zero mode content exceeds the initial one. This follows by the simple fact that the OPE can be computed on the level of modes explicitly by contraction. Thus, it follows that the existence of a well-defined zero mode content puts a natural bound on the maximal zero mode content of OPEs. Therefore, instead of the above inequality (2.16), we then have for the right hand side of the OPE (2.15)

$$
Z_{0}\left(\Psi_{(h ; k)}\right) \leq Z_{0}\left(\Psi_{\left(h_{1} ; k_{1}\right)}\right)+Z_{0}\left(\Psi_{\left(h_{2} ; k_{2}\right)}\right) .
$$

Since, on the other hand, the zero mode content determines the Jordan cell structure via the off-diagonal action of the generators of the chiral symmetry algebra, the inequality (2.17) implies the desired inequality (2.16).

It is clear that we are now faced with a dilemma. If we don't know a priori which fields at which J-levels may contribute to the right hand side of OPEs, we do not have a way to compute the generic form of such OPEs, since we do not have enough information to fix its structure. Thus, we are forced to make even more assumptions on the structure of LCFTs. We will therefore assume throughout the whole paper that a condition of the form (2.16) holds, as long as fields from Jordan cells are concerned. We will justify this assumption as reasonable in section six for the case where the LCFT admits a description in terms of a zero mode content. However, we don't know whether all LCFTs can be described in this way.

As we will briefly discuss at the end of section six, there are indications that LCFTs may not be consistent, if no such condition restricting the maximal J-level of OPEs exists. In general, we don't have any means to compute the structure of arbitrary $n$-point correlation functions in this case, but it is still possible to look at the two-point functions. Our results indicate that these may indeed be inconsistent if, for example, primary members of Jordan cells can produce logarithmic partners in their OPE. 


\section{III. $\quad \operatorname{SL}(2, \mathbb{C})$ COVARIANCE}

IN ORDINARY CFT, two- and three-point functions are determined up to constants which determine the operator algebra and must be fixed by the associativity of the operator product expansion. Moreover, one-point functions are trivial, i.e. $\left\langle\Phi_{h}(z)\right\rangle=\delta_{h, 0}$, although Zamolodchikov pointed out a long time ago, that in non-unitary CFTs, non-vanishing onepoint functions might be possible. For the beginning, we consider only the chiral half of the theory, but keep in mind that LCFTs are known not to factorize entirely into chiral and anti-chiral halfs.

In order to find the generic structure of two- and three-point functions in logarithmic CFTs, we must consider different cases. We start with the simplest setting, as outlined in the preceeding section. Throughout this section we will therefore assume the following: We consider correlation functions of fields $\Psi_{\left(h_{i} ; k_{i}\right)}\left(z_{i}\right)$ from Jordan cells where $\Psi_{\left(h_{i} ; 0\right)}\left(z_{i}\right)$ are proper primary fields. This assumption guarantees that the operator product expansion of two of the primaries will contain only primary fields (and their descendants). Thus, the primaries behave exactly as in an ordinary CFT. Furthermore, we assume that all the logarithmic fields in the Jordan cells are quasi-primary, i.e. that $L_{1} \Psi_{\left(h_{i} ; k_{i}\right)}(0)|0\rangle=0$ for all $k_{i}=0, \ldots, r\left(h_{i}\right)-1$. Such Jordan cells will be called proper Jordan cells in the following. We remark that in any sensible LCFT there is at least one (possibly trivial) Jordan cell which satisfies these assumptions, namely the $h=0$ Jordan cell where the primary is the identity field. The identity should exist in any sensible CFT, since it is the unique field associated with the $S L(2, \mathbb{C})$-invariant vacuum.

Under these assumptions, as shown in the latter two references in [16], the action of the Virasoro modes receives an additional non-diagonal term, namely

$$
\begin{aligned}
& L_{n}\left\langle\Psi_{\left(h_{1} ; k_{1}\right)}\left(z_{1}\right) \ldots \Psi_{\left(h_{n} ; k_{n}\right)}\left(z_{n}\right)\right\rangle= \\
& \sum_{i} z_{i}^{n}\left[z_{i} \partial_{i}+(n+1)\left(h_{i}+\hat{\delta}_{h_{i}}\right)\right]\left\langle\Psi_{\left(h_{1} ; k_{1}\right)}\left(z_{1}\right) \ldots \Psi_{\left(h_{n} ; k_{n}\right)}\left(z_{n}\right)\right\rangle
\end{aligned}
$$

where $n \in \mathbb{Z}$ and the off-diagonal action is $\hat{\delta}_{h_{i}} \Psi_{\left(h_{j} ; k_{j}\right)}(z)=\delta_{i j} \Psi_{\left(h_{j} ; k_{j}-1\right)}(z)$ for $k_{j}>0$ and $\hat{\delta}_{h_{i}} \Psi_{\left(h_{j} ; 0\right)}(z)=0$. This little extension has tremendous consequences. As we are going to show, even the simplest quantities, namely the one-point functions, are severely modified in their behavior. To start with, we recall that only infinitesimal conformal transformations in the algebra $\mathfrak{s l}(2, \mathbb{C})$ can be integrated to global conformal transformation on the Riemann sphere. Thus, only the generators $L_{-1}, L_{0}$, and $L_{1}$ of the Möbius group admit globally valid conservation laws, which usually are expressed in terms of the so-called conformal Ward identities

$$
0=\left\{\begin{aligned}
L_{-1} G\left(z_{1}, \ldots z_{n}\right) & =\sum_{i} \partial_{i} G\left(z_{1}, \ldots z_{n}\right) \\
L_{0} G\left(z_{1}, \ldots z_{n}\right) & =\sum_{i}\left(z_{i} \partial_{i}+h_{i}+\hat{\delta}_{h_{i}}\right) G\left(z_{1}, \ldots z_{n}\right), \\
L_{1} G\left(z_{1}, \ldots z_{n}\right) & =\sum_{i}\left(z_{i}^{2} \partial_{i}+2 z_{i}\left[h_{i}+\hat{\delta}_{h_{i}}\right]\right) G\left(z_{1}, \ldots z_{n}\right),
\end{aligned}\right.
$$

where $G\left(z_{1}, \ldots z_{n}\right)$ denotes an arbitrary $n$-point function $\left\langle\Psi_{\left(h_{1} ; k_{1}\right)}\left(z_{1}\right) \ldots \Psi_{\left(h_{n} ; k_{n}\right)}\left(z_{n}\right)\right\rangle$ of primary fields and/or their logarithmic partner fields. Here, we already have written down the Ward identities in the form valid for proper Jordan cells in logarithmic conformal field theories. We will see in section six, that the assumption of quasi-primary logarithmic fields can be relaxed under certain circumstances. 


\section{III.1. ONE-POINT FunCTIONS}

Let us now apply the Ward identities (3.2) to an arbitrary one-point function $G(z)=$ $\left\langle\Psi_{(h ; k)}(z)\right\rangle$ of a field in a rank $r$ Jordan cell. The identity for $L_{-1}$ states translational invariance such that $G(z)=E_{(h ; k)}$ must be a constant independent of the position $z$. But the identity for $L_{0}$, stating scaling and rotational invariance, leads to the condition

$$
h E_{(h ; k)}+\left(1-\delta_{k, 0}\right) E_{(h ; k-1)}=0 .
$$

In case of the one-point functions, special conformal transformations do not yield an additional constraint. However, the above condition immediately results in the recursive relation, $E_{(h ; r-1-l)}=(-h)^{l} E_{(h ; r-1)}$, such that, if $E_{(h ; r-1)}$ is non-zero, automatically all other one-point functions in this Jordan cell also do not vanish, as long as $h \neq 0$. For $h=0$, the only non-vanishing one-point function is the one of highest possible J-level, i.e. $E_{(h ; r-1)} \neq 0, E_{(h ; k)}=0$ for $0 \leq k<r-1$. Note that $E_{(h=0 ; r-1)}$ must be non-zero. Otherwise, the whole Jordan module to fields of scaling dimension zero could be removed from the theory, since it were orthogonal to all other states. Then, the remaining CFT would not have a vacuum state. To be specific, we from now on normalize $E_{(0 ; r-1)}=1$.

We can learn one more thing from the one-point functions: If a field $\Psi_{(h ; k)}(z), 0<k<$ $r$, were not quasi-primary, special conformal transformations yield a non-zero result as long as the expectation value of the non-quasi-primary contribution is non-zero. Namely,

$$
L_{1}\left\langle\Psi_{(h ; k)}(z)\right\rangle=(-h)^{r-1-k} L_{1} E_{(h ; r-1)}=\left\langle\Psi_{(h-1)}^{\prime}(z)\right\rangle
$$

for a suitable (not necessarily quasi-primary) field $\Psi^{\prime}(z)$ with conformal weight $h-1$ (which is not necessarily part of a Jordan cell, which is why we omit the J-level). We will see in section six that one property of the off-diagonal action of the Virasoro modes is to reduce the zero mode content, i.e. $Z_{0}\left(\Psi^{\prime}\right)<Z_{0}(\Psi)$. Hence, instead of quasi-primarity, a weaker condition will always hold, namely $L_{1}^{n(h, k)} \Psi_{(h ; k)}(0)|0\rangle=0$ for a certain $n(h, k)$ depending on the conformal weight $h$ and the J-level $k$. Moreover, the reduced zero mode content may already force the vacuum expectation value to vanish, i.e. the situation may arise that $L_{1} \Psi_{(h ; k)}(0)|0\rangle \neq 0$, but $L_{1}\left\langle\Psi_{(h ; k)}(z)\right\rangle=0$. This is precisely the case for the example given in section II.1, namely the Jordan cell at $h=1$. In particular, $n(h=1,1)=2$ in the $c=-2$ case, i.e. $L_{1}^{2}|h=1 ; 1\rangle=L_{1}|\xi\rangle=0$ but $\langle\xi\rangle=0$.

\section{III.2. Two-POINT FunCtions}

The next step is to consider two-point functions $G=\left\langle\Psi_{\left(h_{1} ; k_{1}\right)}\left(z_{1}\right) \Psi_{\left(h_{2} ; k_{2}\right)}\left(z_{2}\right)\right\rangle$ of two fields belonging to Jordan cells of ranks $r_{1}, r_{2}$ respectively. Translational invariance tells us that $G=G\left(z_{12}\right)$ is a function of the distance only. Scaling invariance then leads to the ordinary first order differential equation

$$
\left(z_{12} \partial_{z_{12}}+h_{1}+h_{2}\right) G\left(z_{12}\right)+\left\langle\Psi_{\left(h_{1} ; k_{1}-1\right)}\left(z_{1}\right) \Psi_{\left(h_{2} ; k_{2}\right)}\left(z_{2}\right)\right\rangle+\left\langle\Psi_{\left(h_{1} ; k_{1}\right)}\left(z_{1}\right) \Psi_{\left(h_{2} ; k_{2}-1\right)}\left(z_{2}\right)\right\rangle .
$$

The generic solution to this inhomogeneous equation is already surprisingly complicated. Let us introduce some nomenclature to denote where in a correlator logarithmic partners of a primary are inserted by writing

$$
\left\langle\Psi_{\left(h_{1} ; k_{1}\right)}\left(z_{1}\right) \Psi_{\left(h_{2} ; k_{2}\right)}\left(z_{2}\right) \ldots \Psi_{\left(h_{n} ; k_{n}\right)}\left(z_{n}\right)\right\rangle=G_{k_{1}, k_{2}, \ldots k_{n}}\left(z_{1}, z_{2}, \ldots z_{n}\right) .
$$


The above equation then becomes $\left(z_{12} \partial_{z_{12}}+h_{1}+h_{2}\right) G_{k_{1}, k_{2}}\left(z_{12}\right)=-G_{k_{1}-1, k_{2}}\left(z_{12}\right)-$ $G_{k_{1}, k_{2}-1}\left(z_{12}\right)$ with solution

$$
G_{k_{1}, k_{2}}\left(z_{12}\right)=\left(z_{12}\right)^{-h_{1}-h_{2}}\left(D_{\left(h_{1} ; k_{1}\right)\left(h_{2} ; k_{2}\right)}-\int^{z_{12}} \frac{\mathrm{d} \zeta}{\zeta^{1-h_{1}-h_{2}}}\left[G_{k_{1}-1, k_{2}}(\zeta)+G_{k_{1}, k_{2}-1}(\zeta)\right]\right) .
$$

An explicit solution can be found in a hierarchical way, starting with the two-point function of proper primary fields, $G_{0,0}\left(z_{1}, z_{2}\right)$. The conformal Ward identities then reduce to the common CFT case with the well-known solution

$$
\left\langle\Phi_{h_{1}}\left(z_{1}\right) \Phi_{h_{2}}\left(z_{2}\right)\right\rangle=D_{\left(h_{1} ; 0\right)\left(h_{2} ; 0\right)} \delta_{h_{1}, h_{2}}\left(z_{1}-z_{2}\right)^{-h_{1}-h_{2}} .
$$

However, to be consistent with insertion of an OPE, the constant must satisfy $D_{(h ; 0)(h ; 0)}=$ $C_{(h ; 0)(h ; 0)}^{(0 ; 0)} E_{(0 ; 0)}=0$, due to our results on the one-point functions. Hence, $G_{0,0}\left(z_{1}, z_{2}\right)=0$ and, moreover, $E_{(h ; 0)}=0$ for $h \neq 0$ since the form of the two-point function does not admit contributions from other one-point functions. We conclude that the only non-vanishing one-point function of fields in Jordan cells is $\left\langle\Psi_{(0 ; r(0)-1)}\right\rangle$.

The reader should note that in the above reasoning we crucially made use of the assumption that the primary fields are proper, i.e. that their OPE does not contain any logarithmic fields. We will come back to this point later. However, if the identity of the CFT belongs to a Jordan cell, then the assumption that the primary be proper is automatically satisfied. Moreover, as already stressed earlier, it is assumed throughout this section that all logarithmic partner fields are quasi-primary.

We can go on and consider $G_{1,0}\left(z_{1}, z_{2}\right)$ next. The Ward identities now yield an additional term proportional to $G_{0,0}$, which luckily vanishes as just shown. Therefore, we can conclude that $G_{1,0}$ is non-zero, if and only if $E_{(0 ; 1)}$ is non-zero, i.e. if and only if $r-1=1$. Going on in this manner, we finally arrive at the general statement

$$
\left\langle\Psi_{\left(h_{1} ; k\right)}\left(z_{1}\right) \Psi_{\left(h_{2} ; 0\right)}\left(z_{2}\right)\right\rangle=\left\langle\Psi_{\left(h_{1} ; 0\right)}\left(z_{1}\right) \Psi_{\left(h_{2} ; k\right)}\left(z_{2}\right)\right\rangle=\delta_{h_{1}, h_{2}} \delta_{k, r-1} D_{\left(h_{1}, h_{1} ; r-1\right)} \cdot\left(z_{12}\right)^{-2 h_{1}}
$$

which does not depend on which of the two fields is the field of maximal J-level. It is more complicated to compute two-point functions where both fields have J-level larger zero, except when the Jordan rank is $r=2$. Then the only other possibility is $G_{1,1}\left(z_{1}, z_{2}\right)$, where the Ward identities yield contributions proportional to $G_{1,0}=G_{0,1}$ with solution $\left\langle\Psi_{(h ; 1)}\left(z_{1}\right) \Psi_{(h ; 1)}\left(z_{2}\right)\right\rangle=\left(z_{12}\right)^{-2 h}\left[D_{(h, h ; 2)}-2 D_{(h, h ; 1)} \log \left(z_{12}\right)\right]$.

When generalizing to arbitrary rank Jordan cells, the following picture emerges for the two-point functions: The structure constants depend only on the total J-level, i.e. $D_{(h ; k)(h ; l)}=D_{\left(h ; k^{\prime}\right)\left(h ; l^{\prime}\right)} \equiv D_{(h, h ; k+l)}$ for $k+l=k^{\prime}+l^{\prime}$, and they vanish, if the total J-level is less than the rank of the vacuum representation, i.e. $D_{(h ; k)(h ; l)}=0$ for $k+l+1<r(h=0)$. Another consequence is that the only non-vanishing one-point function of type $E_{(h ; k)}$ is $E_{(h=0 ; r(h=0)-1)}$. This, in turn, implies that a logarithmic CFT is only consistently possible, if the vacuum representation is a Jordan cell representation of maximal rank $r(h=0) \geq r(h) \forall h \neq 0$. We then say that the LCFT has rank $r$. Putting things together, the complete solution for the two-point function must have the form

$$
\left\langle\Psi_{\left(h_{1} ; k_{1}\right)}\left(z_{1}\right) \Psi_{\left(h_{2} ; k_{2}\right)}\left(z_{2}\right)\right\rangle=\delta_{h_{1}, h_{2}}\left(\sum_{\ell=0}^{k_{1}+k_{2}} D_{\left(h_{1}, h_{2} ; k_{1}+k_{2}-\ell\right)} \frac{(-2)^{\ell}}{\ell !} \log ^{\ell}\left(z_{12}\right)\right)\left(z_{12}\right)^{-h_{1}-h_{2}}
$$

where we have indicated the implicit condition $h_{1}=h_{2}$ and where for a rank $r$ LCFT all constants $D_{(h, h ; k)}=0$ for $k<r-1$. This result was first obtained in 67. In this 
way, the two-point functions define for each possible conformal weight $h$ matrices $G_{k_{1}, k_{2}}^{(2)}$ of size $r(h) \times r(h)$. However, these matrices depend only on $2 r(h)-r$ yet undetermined constants $D_{(h, h ; k)}, r-1 \leq k \leq 2 r(h)-2$. Moreover, all entries above the anti-diagonal are zero. This last property, i.e. that $D_{(h, h ; k)}=0$ for $k<r-1$, is due to the one-point functions since

$$
\begin{aligned}
D_{(h, h ; k)} & =\frac{1}{2 r(h)-k-1} \sum_{\substack{0 \leq \ell_{1}, \ell_{2} \leq r(h)-1 \\
\ell_{1}+\ell_{2}=k}} C_{\left(h ; \ell_{1}\right)\left(h ; \ell_{2}\right)}^{(0 ; r-1)} E_{(0 ; r-1)} \\
& = \begin{cases}C_{(h, h, 0 ; k+r-1)} & \text { for } r-1 \leq k<2 r-1, \\
0 & \text { else. }\end{cases}
\end{aligned}
$$

Note that the three-point structure constants do, in effect, only depend on the total Jlevel, as we have tried to indicate in our notation. The special form of the two-point structure constant matrices ensures that they are always invertible.

It is often very convenient to work with states instead of the fields directly, in particular when purely algebraic properties such as null states are considered. As usual, we have an isomorphism between the space of fields and the space of states furnished by the map $|h ; k\rangle=\Psi_{(h ; k)}(0)|0\rangle$. Although one does not necessarily have a scalar product on the space of states, one can introduce a pairing, the Shapovalov form, between states and linear functionals. Identifying the out-states with (a subset of) the linear functionals equips the space of states with a Hilbert space like structure. As in ordinary conformal field theory, we have $\langle h ; k|=(|h ; k\rangle)^{\dagger}=\lim _{z \rightarrow 0}\langle 0| \Psi_{(h ; k)}(1 / z)$. Using now that logarithmic fields transform under conformal mappings $z \mapsto f(z)$ as

$$
\begin{aligned}
\Psi_{(h ; k)}(z) & =\sum_{l=0}^{k} \frac{1}{l !} \frac{\partial^{l}}{\partial h^{l}}\left(\frac{\partial f(z)}{\partial z}\right)^{h} \Psi_{(h ; k-l)}(f(z)) \\
& =\sum_{l=0}^{k} \frac{1}{l !} \log ^{l}\left|\frac{\partial f(z)}{\partial z}\right|\left(\frac{\partial f(z)}{\partial z}\right)^{h} \Psi_{(h ; k-l)}(f(z)),
\end{aligned}
$$

the out-state can be re-expressed in a form which allows us to apply (3.9) to evaluate the Shapovalov form. In ordinary conformal field theory, we simply get $\langle h|=$ $\lim _{z \rightarrow \infty}\langle 0| z^{2 h} \Psi_{h}(z)$ such that $\left\langle h \mid h^{\prime}\right\rangle=\delta_{h, h^{\prime}}$ up to normalization. Interestingly, the transformation behavior of logarithmic fields yields a very similar result, canceling all logarithmic divergences. Thus, we obtain for the Shapovalov form

$$
\left\langle h ; k \mid h^{\prime} ; k^{\prime}\right\rangle=\delta_{h ; h^{\prime}} D_{\left(h, h^{\prime} ; k+k^{\prime}\right)},
$$

which is a lower triangular matrix. To demonstrate this, we consider the example of a rank two LCFT. Then we clearly have $\langle h ; 0 \mid h ; 0\rangle=0,\langle h ; 1 \mid h ; 0\rangle=\langle h ; 0 \mid h ; 1\rangle=D_{(h, h ; 1)}$ and with

$$
\lim _{z \rightarrow 0}\left\langle 0 \mid \Psi_{(h ; 1)}(1 / z) \Psi_{(h ; 1)}(0)\right\rangle=\lim _{z \rightarrow \infty}\left\langle 0\left|z^{2 h}\left[\Psi_{(h ; 1)}(z)+2 \log (z) \Psi_{(h ; 0)}(z)\right] \Psi_{(h ; 1)}(0)\right| 0\right\rangle
$$

the desired result $\langle h ; 1 \mid h ; 1\rangle=D_{(h, h ; 2)}$. Hence, the Shapovalov form is well defined and non-degenerate for the logarithmic case much in the same way as it can be defined for ordinary CFTs. Note that the definition of the Shapovalov form does not depend on whether the CFT is unitary or not. 
For completeness, we mention that the Shapovalov form is not uniquely defined in LCFTs, because the basis $\{|h ; k\rangle: k=0, \ldots r(h)-1\}$ of states is not unique. The reason is that we always have the freedom to redefine the logarithmic partner fields, or their states respectively, as

$$
\Psi_{(h ; k)}^{\prime}(z)=\Psi_{(h ; k)}(z)+\sum_{i=1}^{k} \lambda_{i} \Psi_{(h ; k-i)}(z)
$$

with arbitrary constants $\lambda_{i}$. At this state, there are no further restrictions from the structure of the LCFT which could fix a basis within the Jordan cells. Only the proper primary field, or the proper highest-weight state respectively, is uniquely defined up to normalization.

\section{III.3. ThrEe-POINT FunCtIONS}

The three-point functions can be fixed along the same lines, although the procedure is now more complicated. For each triplet $h_{1}, h_{2}, h_{3}$ of conformal weights, we find a set of $r\left(h_{1}\right) \times r\left(h_{2}\right) \times r\left(h_{3}\right)$ functions $G_{k_{1}, k_{2}, k_{3}}\left(z_{1}, z_{2}, z_{3}\right)$. From now on we will restrict ourselves to the case where $r(h)=r$ for all Jordan cells in the LCFT. We will see shortly that otherwise no consistent definition of OPEs seems possible. With this restriction, we can collect the set of three-point functions into $r$ matrices, each of size $r \times r$, namely the matrices $\left(G_{k_{1}}^{(3)}\right)_{k_{2}, k_{3}}$.

A closed formula of the type as given above for the two-point function is extremely lengthy. However, the three-point functions can all be given in the form:

$$
\begin{gathered}
\left\langle\Psi_{\left(h_{1} ; k_{1}\right)} \Psi_{\left(h_{2} ; k_{2}\right)} \Psi_{\left(h_{3} ; k_{3}\right)}\right\rangle=\sum_{k=r-1}^{k_{1}+k_{2}+k_{3}} C_{\left(h_{1}, h_{2}, h_{3} ; k\right)} \sum_{j_{1}=0}^{k_{1}} \sum_{j_{2}=0}^{k_{2}} \sum_{j_{3}=0}^{k_{3}} \delta_{j_{1}+j_{2}+j_{3}, k_{1}+k_{2}+k_{3}-k} \\
\times \frac{1}{j_{1} ! j_{2} ! j_{3} !}\left(\partial_{h_{1}}\right)^{j_{1}}\left(\partial_{h_{2}}\right)^{j_{2}}\left(\partial_{h_{3}}\right)^{j_{3}}\left(z_{12}^{h_{3}-h_{1}-h_{2}} z_{13}^{h_{2}-h_{1}-h_{3}} z_{23}^{h_{1}-h_{2}-h_{3}}\right) .
\end{gathered}
$$

The corresponding formula for the two-point function can be rewritten in the same manner involving derivatives with respect to the conformal weight,

$$
\left\langle\Psi_{\left(h_{1} ; k_{1}\right)} \Psi_{\left(h_{2} ; k_{2}\right)}\right\rangle=\sum_{k=r-1}^{k_{1}+k_{2}} \delta_{h_{1}, h_{2}} D_{\left(h_{1}, h_{2} ; k_{1}+k_{2}-k\right)} \frac{1}{k !}\left(\partial_{h_{2}}\right)^{k}\left(z_{12}\right)^{-2 h_{2}},
$$

which evaluates to exactly the form given in (3.9). Note that again the yet free structure constants depend only on the total J-level. This agrees with what one might expect from the total symmetry of the three-point structure constants under permutations. Differentiation with respect to the conformal weights reproduces precisely the logarithmic contributions to satisfy the inhomogeneous Ward identities.

These expressions can be made even more suggestive, if one treats the structure constants as (analytic) functions of the conformal weights 67. This is actually true in the case of minimal models, where all structure constants can be given explicitly as functions of the charges within a free field representations, and hence in terms of the conformal weights. Putting simply

$$
C_{\left(h_{1}, h_{2}, h_{3} ; k+r-1\right)}=\frac{1}{k !} \sum_{i_{1}+i_{2}+i_{3}=k-(r-1)}\left(\partial_{h_{1}}\right)^{i_{1}}\left(\partial_{h_{2}}\right)^{i_{2}}\left(\partial_{h_{2}}\right)^{i_{2}} C_{h_{1}, h_{2}, h_{3}},
$$

allows to rewrite (3.11) entirely in terms of derivatives with respect to the conformal weights. Here, $C_{\left(h_{1}, h_{2}, h_{3} ; r-1\right)}$ is then the pure, not differentiated, structure constant. 


\section{Operator Product Expansions}

With THE COMPlETE set of two- and three-point functions at hand, we can now proceed to determine the operator product expansions in their generic form. To do this, we

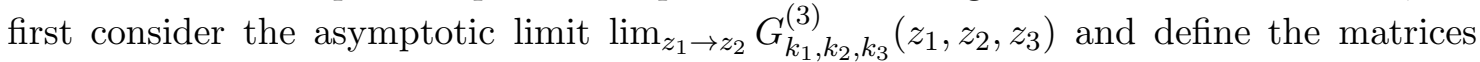
$\left(G_{k_{1}}^{(3)}\right)_{k_{2}, k_{3}}$ in this limit. This essentially amounts to replacing $z_{13}$ by $z_{23}$. Next, we take the two-point functions $G_{k_{1}, k_{2}}^{(2)}\left(z_{2}, z_{3}\right)$, collect them into a matrix $\left(G^{(2)}\right)_{k_{1}, k_{2}}$ and invert the latter to obtain $\left(G^{(2)}\right)^{\ell_{1}, \ell_{2}}$. Finally, the matrix product

$$
C_{\left(h_{1}, h_{2} ; k_{1}+k_{2}\right)}^{\left(h_{3} ; k_{3}\right)}=\left(G_{k_{1}}^{(3)}\right)_{k_{2}, k}\left(G^{(2)}\right)^{k, k_{3}}
$$

yields matrices $\left(C_{\left(h_{1} ; k_{1}\right), h_{2}}^{h_{3}}\right)_{k_{2}}^{k_{3}}$ encoding all the OPEs of the field $\Psi_{\left(h_{1} ; k_{1}\right)}(z)$ with fields of arbitrary J-level. An immediate consequence of (4.1) is now that associativity of the operator algebra can only hold if the rank of all Jordan cells is equal. Indeed, assuming the contrary, the matrices $\left(G_{k}^{(3)}\right)_{l m}$ were not always square matrices, and the rank of the matrices $\left(G^{(2)}\right)^{k l}$ would depend on the conformal weight. It is now easy to see that the associativity conditions such as crossing symmetry

$$
C_{\left(h_{i}, h_{j} ; k_{i}+k_{j}\right)}^{(h ; k)} C_{\left(h, h_{l}, h_{m} ; k+k_{l}+k_{m}\right)}=C_{\left(h_{i}, h_{l} ; k_{i}+k_{l}\right)}^{\left(h^{\prime} ; k^{\prime}\right)} C_{\left(h^{\prime}, h_{j}, h_{m} ; k^{\prime}+k_{j}+k_{m}\right)}
$$

cannot any longer hold, since the matrices on both sides of the equation were not always of equal rank. In effect, associativity can only be kept if the ranks of the Jordan cells appearing implicitly on both sides of the equation can consistently be restricted to the minimal rank of the product matrices. This minimal rank will automatically define the maximal rank of the LCFT under consideration. This justifies our earlier restriction.

To see, how this formula works, we will give a more explicit version of (4.1). Let us denote the complete set of two-point functions as $\langle\ell, k\rangle=G_{\ell, k}^{(2)}\left(z_{2}, z_{3}\right)=\left\langle\Psi_{(h ; \ell)}\left(z_{2}\right) \Psi_{(h ; k)}\left(z_{3}\right)\right\rangle$ and correspondingly the three-point functions as $\left\langle\ell, k_{1}, k_{2}\right\rangle=\lim _{z_{1} \rightarrow z_{2}} G_{k_{1}, k_{2}, \ell}^{(3)}\left(z_{1}, z_{2}, z_{3}\right)=$ $\lim _{z_{1} \rightarrow z_{2}}\left\langle\Psi_{\left(h_{1} ; k_{1}\right)}\left(z_{1}\right) \Psi_{\left(h_{2} ; k_{2}\right)}\left(z_{2}\right) \Psi_{(h ; \ell)}\left(z_{3}\right)\right\rangle$, all essentially given by formulae (3.9) and (3.11). The reader should not confuse this notation with the notation for the Shapovalov form introduced earlier. Then, the OPEs take the structure

$$
\begin{aligned}
& \Psi_{h_{1} ; k_{1}}\left(z_{1}\right) \Psi_{h_{2} ; k_{2}}\left(z_{2}\right)=\sum_{h} \sum_{k=0}^{r-1}\left(\prod_{i=0}^{r-1}\langle i, r-1-i\rangle\right)^{-1} \\
& \times\left|\begin{array}{ccccccc}
\langle 0,0\rangle & \ldots & \langle 0, k-1\rangle & \left\langle 0, k_{1}, k_{2}\right\rangle & \langle 0, k+1\rangle & \ldots & \langle 0, r-1\rangle \\
\vdots & \ddots & \vdots & \vdots & \vdots & \ddots & \vdots \\
\langle\ell, 0\rangle & \ldots & \langle\ell, k-1\rangle & \left\langle\ell, k_{1}, k_{2}\right\rangle & \langle\ell, k+1\rangle & \ldots & \langle\ell, r-1\rangle \\
\vdots & \ddots & \vdots & \vdots & \vdots & \ddots & \\
\langle r-1,0\rangle & \ldots & \langle r-1, k-1\rangle & \left\langle r-s, k_{1}, k_{2}\right\rangle & \langle r-1, k+1\rangle & \ldots & \langle r-1, r-1\rangle
\end{array}\right| \Psi_{(h ; k)}\left(z_{2}\right),
\end{aligned}
$$

which in passing also proves that the matrix of two-point functions can be inverted without problems. Of course, the denominator is written here in a particularly symmetric way, it equals $\langle j, r-1-j\rangle^{r}$ for any $0 \leq j \leq r-1$. Note that the only non-zero entries above the anti-diagonal stem from the inserted column of three-point functions. The formula (4.1) or (4.3) respectively are the sought after generalization of (1.3) to the case of logarithmic CFTs. 
With this result, we obtain in the simplest $r=2$ case the well known OPEs

$$
\begin{aligned}
& \Psi_{\left(h_{1} ; 0\right)}(z) \Psi_{\left(h_{2} ; 0\right)}(0)=\sum_{h} \frac{C_{\left(h_{1}, h_{2}, h ; 1\right)}}{D_{(h, h ; 1)}} \Psi_{(h ; 0)}(0) z^{h-h_{1}-h_{2}}, \\
& \Psi_{\left(h_{1} ; 0\right)}(z) \Psi_{\left(h_{2} ; 1\right)}(0)=\sum_{h}\left[\frac{C_{\left(h_{1}, h_{2}, h ; 1\right)}}{D_{(h, h ; 1)}} \Psi_{(h ; 1)}(0)\right. \\
& \left.+\frac{D_{(h, h ; 1)} C_{\left(h_{1}, h_{2}, h ; 2\right)}-D_{(h, h ; 2)} C_{\left(h_{1}, h_{2}, h ; 1\right)}}{D_{(h, h ; 1)}^{2}} \Psi_{(h ; 0)}(0)\right] z^{h-h_{1}-h_{2}}, \\
& \Psi_{\left(h_{1} ; 1\right)}(z) \Psi_{\left(h_{2} ; 1\right)}(0)=\sum_{h}\left[\left(\frac{C_{\left(h_{1}, h_{2}, h ; 2\right)}}{D_{(h, h ; 1)}}-\frac{2 C_{\left(h_{1}, h_{2}, h ; 1\right)}}{D_{(h, h ; 1)}} \log (z)\right) \Psi_{(h ; 1)}(0)\right. \\
& +\left(\frac{D_{(h, h ; 1)} C_{\left(h_{1}, h_{2}, h ; 3\right)}-D_{(h, h ; 2)} C_{\left(h_{1}, h_{2}, h ; 2\right)}}{D_{(h, h ; 1)}^{2}}\right. \\
& +\frac{2 D_{(h, h ; 2)} C_{\left(h_{1}, h_{2}, h ; 1\right)}-D_{(h, h ; 1)} C_{\left(h_{1}, h_{2}, h ; 2\right)}}{D_{(h, h ; 1)}^{2}} \log (z) \\
& \left.\left.-\frac{D_{(h, h ; 1)} C_{\left(h_{1}, h_{2}, h ; 1\right)}}{D_{(h, h ; 1)}^{2}} \log ^{2}(z)\right) \Psi_{(h ; 0)}(0)\right] z^{h-h_{1}-h_{2}}
\end{aligned}
$$

Note that, for instance, the OPE of a proper primary with its logarithmic partner necessarily receives two contributions. One might naively have expected that proper primary fields do not change the J-level, although already the OPE of the stress-energy tensor with a logarithmic field will have an additional term involving the primary field. At the end of this section we will give a complete non-trivial example, namely the full set of generic OPE forms for a LCFT with rank four Jordan cells.

But before doing so, we want to remark on the question of locality. The two- and three-point functions and the OPEs can easily be brought into a form for a local LCFT constructed out of left- and right-chiral half. The rule for this is simply to replace each $\log \left(z_{i j}\right)$ by $\log \left|z_{i j}\right|^{2}$, and to replace each power $\left(z_{i j}\right)^{\mu_{i j}}$ by $\left|z_{i j}\right|^{2 \mu_{i j}}$. This yields a LCFT where all fields have the same holomorphic and anti-holomorphic scaling dimensions and the same J-level. Such an ansatz automatically satisfies both, the holomorphic as well as the anti-holomorphic Ward identities, if $z$ and $\bar{z}$ are formally treated as independent variables. It is important to note, however, that the resulting full amplitudes do not factorize into holomorphic and anti-holomorphic parts. This is a well known feature of LCFTs. For example, the OPE equation (4.6) would read in its full form

$$
\begin{aligned}
& \Psi_{\left(h_{1} ; 1\right)}(z, \bar{z}) \Psi_{\left(h_{2} ; 1\right)}(0,0)=\sum_{h}|z|^{2\left(h-h_{1}-h_{2}\right)}\left[\frac{C_{(2)}-2 C_{(1)} \log |z|^{2}}{D_{(1)}} \Psi_{(h ; 1)}(0,0)\right. \\
& \left.+\left(\frac{D_{(1)} C_{(3)}-D_{(2)} C_{(2)}}{D_{(1)}^{2}}+\frac{2 D_{(2)} C_{(1)}-D_{(1)} C_{(2)}}{D_{(1)}^{2}} \log |z|^{2}-\frac{D_{(1)} C_{(1)}}{D_{(1)}^{2}} \log ^{2}|z|^{2}\right) \Psi_{(h ; 0)}(0,0)\right]
\end{aligned}
$$

with an obvious abbreviation for the structure constants. The reader is encouraged to convince herself of both, that on one hand this does indeed not factorize into holomorphic and anti-holomorphic parts, but that on the other hand this does satisfy the full set of conformal Ward identities. 


\section{IV.1. A Non-trivial Example}

Now, we wish to present a fully worked out non-trivial example in order to demonstrate that even the generic structure of OPEs in arbitrary rank LCFTs is indeed more complicated than naively thought. Therefore, we present the OPEs for a rank four LCFT. Although all explicitly known LCFTs such as $c=-2$ and all the other $c_{p, 1}$ models [27, 16], or certain non-trivial $c=0$ models 29, 30] are only rank two LCFTs, there are many indications that higher rank LCFTs exist. For instance, null-vectors for higher rank LCFTs have been noted in the latter two references in [16], and general considerations on higher rank LCFTs have been made in [23, 26]. Examples were found in the first work in [9] and in [56]. As a rule of thumb, one can reasonably conjecture that a CFT with a degenerate vacuum structure due to the existence of non-trivial zero-modes can be extended to a logarithmic CFT, whose maximal J-level (i.e. $r-1$ ) precisely equals the number of zero-modes (or of anti-commuting pairs of zero modes). Again, $c=-2$ is here the prime example, since the well known $b c$ system of conformal spins 1 and 0 can indeed be expressed in terms of the $\theta_{+} \theta_{-}$system briefly mentioned in section II.1. The $c=-2$ ghost system has one crucial zero-mode such that $\langle 0 \mid 0\rangle=0,\left\langle 0\left|c_{0}\right| 0\right\rangle \neq 0$. With the identification $c(z)=\theta_{+}(z), b(z)=\partial \theta_{-}(z)$, the $\theta_{+} \theta_{-}$system on one hand reproduces as a subset all the correlators of the $b c$ system when evaluated sandwiched between $\left\langle\xi_{-}\right|$and $|0\rangle$, and on the other hand constitutes an enlarged CFT which contains logarithmic fields. As discussed above, this CFT is logarithmic of rank two, as we would expect from the number of zero-modes. Work in this direction will appear elsewhere [20].

In order to keep the formulae readable, we will skip all the factors $\left(z_{12}\right)^{h-h_{1}-h_{2}}$ as well as all arguments of the fields. Moreover, we only denote the J-levels in the structure constants omitting all references to the conformal weights. Hence, we put $C_{k} \equiv C_{\left(h_{1}, h_{2}, h ; k\right)}$ and $D_{k} \equiv D_{\left(h_{2}, h ; k\right)}$. Furthermore, $\ell^{k}$ is a shorthand for $\log ^{k}\left(z_{12}\right)$.

$$
\begin{aligned}
\Psi_{\left(h_{1} ; 0\right)} \Psi_{\left(h_{2} ; 0\right)} & =\boldsymbol{\Sigma}_{h} \frac{C_{3}}{D_{3}} \Psi_{(h ; 0)}, \\
\Psi_{\left(h_{1} ; 0\right)} \Psi_{\left(h_{2} ; 1\right)} & =\boldsymbol{\Sigma}_{h} \frac{C_{3}}{D_{3}} \Psi_{(h ; 1)}+\frac{C_{4} D_{3}-C_{3} D_{4}}{D_{3}^{2}} \Psi_{(h ; 0)}, \\
\Psi_{\left(h_{1} ; 0\right)} \Psi_{\left(h_{2} ; 2\right)} & =\boldsymbol{\Sigma}_{h} \frac{C_{3}}{D_{3}} \Psi_{(h ; 2)}+\frac{C_{4} D_{3}-C_{3} D_{4}}{D_{3}^{2}} \Psi_{(h ; 1)}+\frac{C_{5} D_{3}^{2}-C_{4} D_{4} D_{3}+C_{3}\left(D_{4}^{2}-D_{5} D_{3}\right)}{D_{3}^{3}} \Psi_{(h ; 0)}, \\
\Psi_{\left(h_{1} ; 0\right)} \Psi_{\left(h_{2} ; 3\right)} & =\boldsymbol{\Sigma}_{h} \frac{C_{3}}{D_{3}} \Psi_{(h ; 3)}+\frac{C_{4} D_{3}-C_{3} D_{4}}{D_{3}^{2}} \Psi_{(h ; 2)}+\frac{C_{5} D_{3}^{2}-C_{4} D_{4} D_{3}+C_{3}\left(D_{4}^{2}-D_{5} D_{3}\right)}{D_{3}^{3}} \Psi_{(h ; 1)} \\
& +\frac{C_{6} D_{3}^{3}-C_{5} D_{4} D_{3}^{2}+C_{4}\left(D_{4}^{2}-D_{5} D_{3}\right) D_{3}-C_{3}\left(D_{6} D_{3}^{2}-2 D_{5} D_{4} D_{3}+D_{4}^{3}\right)}{D_{3}^{4}} \Psi_{(h ; 0)}, \\
& =\boldsymbol{\Sigma}_{h} \frac{C_{3}}{D_{3}} \Psi_{(h ; 2)}+\left(\frac{C_{4} D_{3}-C_{3} D_{4}}{D_{3}^{2}}-\frac{C_{3}}{D_{3}} \ell\right) \Psi_{(h ; 1)} \\
\Psi_{\left(h_{1} ; 1\right)} \Psi_{\left(h_{2} ; 1\right)} & \left(\frac{C_{5} D_{3}^{2}-C_{4} D_{4} D_{3}+C_{3}\left(D_{4}^{2}-D_{5} D_{3}\right)}{D_{3}^{3}}-\frac{C_{4} D_{3}-C_{3} D_{4}}{D_{3}^{2}} \ell-\frac{C_{3}}{2 D_{3}} \ell^{2}\right) \Psi_{(h ; 0)}, \\
\Psi_{\left(h_{1} ; 1\right)} \Psi_{\left(h_{2} ; 2\right)} & \boldsymbol{\Sigma}_{h} \frac{C_{3}}{D_{3}} \Psi_{(h ; 3)}+\left(\frac{C_{4} D_{3}-C_{3} D_{4}}{D_{3}^{2}}-\frac{C_{3}}{D_{3}} \ell\right) \Psi_{(h ; 2)} \\
& +\left(\frac{C_{5} D_{3}^{2}-C_{4} D_{4} D_{3}+C_{3}\left(D_{4}^{2}-D_{5} D_{3}\right)}{D_{3}^{3}}-\frac{C_{4} D_{3}-C_{3} D_{4}}{D_{3}^{2}} \ell\right) \Psi_{(h ; 1)} \\
& +\left(\frac{C_{6} D_{3}^{3}-C_{5} D_{4} D_{3}^{2}+C_{4}\left(D_{4}^{2}-D_{5} D_{3}\right) D_{3}-C_{3}\left(D_{6} D_{3}^{2}-2 D_{5} D_{4} D_{3}+D_{4}^{3}\right)}{D_{3}^{4}}\right.
\end{aligned}
$$




$$
\begin{aligned}
& \left.-\frac{C_{5} D_{3}^{2}-C_{4} D_{4} D_{3}+C_{3}\left(D_{4}^{2}-D_{5} D_{3}\right)}{D_{3}^{3}} \ell+\frac{C_{3}}{6 D_{3}} \ell^{3}\right) \Psi_{(h ; 0)}, \\
& \Psi_{\left(h_{1} ; 1\right)} \Psi_{\left(h_{2} ; 3\right)}=\boldsymbol{\Sigma}_{h}\left(\frac{C_{4}}{D_{3}}-\frac{2 C_{3}}{D_{3}} \ell\right) \Psi_{(h ; 3)}+\left(\frac{C_{5} D_{3}-C_{4} D_{4}}{D_{3}^{2}}-\frac{C_{4} D_{3}-2 C_{3} D_{4}}{D_{3}^{2}} \ell-\frac{C_{3}}{2 D_{3}} \ell^{2}\right) \Psi_{(h ; 2)} \\
& +\left(\frac{C_{6} D_{3}^{2}-C_{5} D_{4} D_{3}+C_{4}\left(D_{4}^{2}-D_{5} D_{3}\right)}{D_{3}^{3}}\right. \\
& \left.-\frac{C_{5} D_{3}^{2}-C_{4} D_{4} D_{3}+2 C_{3}\left(D_{4}^{2}-D_{5} D_{3}\right)}{D_{3}^{3}} \ell+\frac{C_{3} D_{4}}{2 D_{3}^{2}} \ell^{2}-\frac{C_{3}}{6 D_{3}} \ell^{3}\right) \Psi_{(h ; 1)} \\
& +\left(\frac{C_{7} D_{3}^{3}-C_{6} D_{4} D_{3}^{2}+C_{5}\left(D_{4}^{2}-D_{5} D_{3}\right) D_{3}-C_{4}\left(D_{6} D_{3}^{2}-2 D_{5} D_{4} D_{3}+D_{4}^{3}\right)}{D_{3}^{4}}\right. \\
& -\frac{C_{6} D_{3}^{3}-C_{5} D_{4} D_{3}^{2}+C_{4}\left(D_{4}^{2}-D_{5} D_{3}\right)-2 C_{3}\left(D_{6} D_{3}^{2}-2 D_{5} D_{4} D_{3}+D_{4}^{3}\right)}{D_{3}^{4}} \ell \\
& \left.-\frac{C_{3}\left(D_{4}^{2}-D_{5} D_{3}\right)}{2 D_{3}^{3}} \ell^{2}+\frac{C_{3} D_{4}}{6 D_{3}^{2}} \ell^{3}-\frac{C_{3}}{12 D_{3}} \ell^{4}\right) \Psi_{(h ; 0)} \\
& \Psi_{\left(h_{1} ; 2\right)} \Psi_{\left(h_{2} ; 2\right)}=\Sigma_{h}\left(\frac{C_{4}}{D_{3}}-\frac{2 C_{3}}{D_{3}} \ell\right) \Psi_{(h ; 3)}+\left(\frac{C_{5} D_{3}-C_{4} D_{4}}{D_{3}^{2}}-\frac{C_{4} D_{3}-2 C_{3} D_{4}}{D_{3}^{2}} \ell\right) \Psi_{(h ; 2)} \\
& +\left(\frac{C_{6} D_{3}^{2}-C_{5} D_{4} D_{3}+C_{4}\left(D_{4}^{2}-D_{5} D_{3}\right)}{D_{3}^{3}}\right. \\
& \left.-\frac{C_{5} D_{3}^{2}-C_{4} D_{4} D_{3}+2 C_{3}\left(D_{4}^{2}-D_{5} D_{3}\right)}{D_{3}^{3}} \ell+\frac{C_{4}}{2 D_{3}} \ell^{2}\right) \Psi_{(h ; 1)} \\
& +\left(\frac{C_{7} D_{3}^{3}-C_{6} D_{4} D_{3}^{2}+C_{5}\left(D_{4}^{2}-D_{5} D_{3}\right) D_{3}-C_{4}\left(D_{6} D_{3}^{2}-2 D_{5} D_{4} D_{3}+D_{4}^{3}\right)}{D_{3}^{4}}\right. \\
& -\frac{C_{6} D_{3}^{3}-C_{5} D_{4} D_{3}^{2}+C_{4}\left(D_{4}^{2}-D_{5} D_{3}\right)-2 C_{3}\left(D_{6} D_{3}^{2}-2 D_{5} D_{4} D_{3}+D_{4}^{3}\right)}{D_{3}^{4}} \ell \\
& \left.+\frac{C_{5} D_{3}-C_{4} D_{4}}{2 D_{3}^{2}} \ell^{2}+\frac{C_{4}}{6 D_{3}} \ell^{3}-\frac{C_{3}}{12 D_{3}} \ell^{4}\right) \Psi_{(h ; 0)}, \\
& \Psi_{\left(h_{1} ; 2\right)} \Psi_{\left(h_{2} ; 3\right)}=\boldsymbol{\Sigma}_{h}\left(\frac{C_{5}}{D_{3}}-\frac{2 C_{4}}{D_{3}} \ell+\frac{2 C_{3}}{D_{3}} \ell^{2}\right) \Psi_{(h ; 3)} \\
& +\left(\frac{C_{6} D_{3}-C_{5} D_{4}}{D_{3}^{2}}-\frac{C_{5} D_{3}-2 C_{4} D_{4}}{D_{3}^{2}} \ell-\frac{2 C_{3} D_{4}}{D_{3}^{2}} \ell^{2}+\frac{5 C_{3}}{6 D_{3}} \ell^{3}\right) \Psi_{(h ; 2)} \\
& +\left(\frac{C_{7} D_{3}^{2}-C_{6} D_{4} D_{3}+D_{5}\left(D_{4}^{2}-D_{5} D_{3}\right)}{D_{3}^{3}}-\frac{C_{6} D_{3}^{2}-C_{5} D_{4} D_{3}+2 C_{4}\left(D_{4}^{2}-D_{5} D_{3}\right)}{D_{3}^{3}} \ell\right. \\
& \left.+\frac{C_{5} D_{3}^{2}+4 C_{3}\left(D_{4}^{2}-D_{5} D_{3}\right)}{2 D_{3}^{3}} \ell^{2}-\frac{C_{4} D_{3}+5 C_{3} D_{4}}{6 D_{3}^{2}} \ell^{3}+\frac{C_{3}}{4 D_{3}} \ell^{4}\right) \Psi_{(h ; 1)} \\
& +\left(\frac{C_{8} D_{3}^{3}-C_{7} D_{4} D_{3}^{2}+C_{6}\left(D_{4}^{2}-D_{5} D_{3}\right) D_{3}-C_{5}\left(D_{6} D_{3}^{2}-2 D_{5} D_{4} D_{3}+D_{4}^{3}\right)}{D_{3}^{4}}\right. \\
& -\frac{C_{7} D_{3}^{3}-C_{6} D_{4} D_{3}^{2}+C_{5}\left(D_{4}^{2}-D_{5} D_{3}\right) D_{3}-2 C_{4}\left(D_{6} D_{3}^{2}-2 D_{5} D_{4} D_{3}+D_{4}^{3}\right)}{D_{3}^{4}} \ell \\
& +\frac{C_{6} D_{3}^{3}-C_{5} D_{4} D_{3}^{2}-4 C_{3}\left(D_{6} D_{3}^{2}-2 D_{5} D_{4} D_{3}+D_{4}^{3}\right)}{2 D_{3}^{4}} \ell^{2} \\
& \left.+\frac{C_{4} D_{4} D_{3}+5 C_{3}\left(D_{4}^{2}-D_{5} D_{3}\right)}{6 D_{3}^{3}} \ell^{3}-\frac{C_{4} D_{3}+3 C_{3} D_{4}}{12 D_{3}^{2}} \ell^{4}+\frac{C_{3}}{12 D_{3}} \ell^{5}\right) \Psi_{(h ; 0)},
\end{aligned}
$$




$$
\begin{aligned}
\Psi_{\left(h_{1} ; 3\right)} \Psi_{\left(h_{2} ; 3\right)}= & \Sigma_{h}\left(\frac{C_{6}}{D_{3}}-\frac{2 C_{5}}{D_{3}} \ell+\frac{2 C_{4}}{D_{3}} \ell^{2}-\frac{4 C_{3}}{3 D_{3}} \ell^{3}\right) \Psi_{(h ; 3)}+\left(\frac{C_{7} D_{3}-C_{6} D_{4}}{D_{3}^{2}}\right. \\
& \left.-\frac{C_{6} D_{3}-2 C_{5} D_{4}}{D_{3}^{2}} \ell-\frac{2 C_{4} D_{4}}{D_{3}^{2}} \ell^{2}+\frac{2 C_{4} D_{3}+4 C_{3} D_{4}}{3 D_{3}^{2}} \ell^{3}-\frac{3 C_{3}}{4 D_{3}} \ell^{4}\right) \Psi_{(h ; 2)} \\
+ & \left(\frac{C_{8} D_{3}^{2}-C_{7} D_{4} D_{3}+C_{6}\left(D_{4}^{2}-D_{5} D_{3}\right)}{D_{3}^{3}}-\frac{C_{7} D_{3}^{2}-C_{6} D_{4} D_{3}+2 C_{5}\left(D_{4}^{2}-D_{5} D_{3}\right)}{D_{3}^{3}} \ell\right. \\
& +\frac{C_{6} D_{3}^{2}+4 C_{4}\left(D_{4}^{2}-D_{5} D_{3}\right)}{2 D_{3}^{3}} \ell^{2}-\frac{C_{5} D_{3}^{2}+2 C_{4} D_{4} D_{3}+4 C_{3}\left(D_{4}^{2}-D_{3} D_{5}\right)}{3 D_{3}^{3}} \ell^{3} \\
& \left.+\frac{C_{4} D_{3}+3 C_{3} D_{4}}{4 D_{3}^{2}} \ell^{4}-\frac{C_{3}}{4 D_{3}} \ell^{5}\right) \Psi_{(h ; 1)} \\
+ & \left(\frac{C_{9} D_{3}^{3}-C_{8} D_{3}^{2} D_{4}+C_{7}\left(D_{4}^{2}-D_{5} D_{3}\right) D_{3}-C_{6}\left(D_{6} D_{3}^{2}-2 D_{5} D_{4} D_{3}+D_{4}^{2}\right)}{D_{3}^{4}}\right. \\
& -\frac{C_{8} D_{3}^{2}-C_{7} D_{4} D_{3}^{2}+C_{6}\left(D_{4}^{2}-D_{5} D_{3}\right) D_{3}-2 C_{5}\left(D_{6} D_{3}^{2}-2 D_{5} D_{4} D_{3}+D_{4}^{3}\right)}{D_{3}^{4}} \ell \\
& +\frac{C_{7} D_{3}^{3}-C_{6} D_{4} D_{3}^{2}-4 C_{4}\left(D_{6} D_{3}^{2}-2 D_{5} D_{4} D_{3}+D_{4}^{3}\right)}{2 D_{3}^{4}} \ell^{2} \\
& -\frac{C_{6} D_{3}^{3}-2 C_{5} D_{4} D_{3}^{2}-4 C_{4}\left(D_{4}^{2}-D_{5} D_{3}\right)-8 C_{3}\left(D_{6} D_{3}^{2}-2 D_{5} D_{4} D_{3}+D_{4}^{3}\right)}{6 D_{3}^{4}} \ell^{3} \\
& \left.-\frac{C_{5} D_{3}^{2}+3 C_{4} D_{4} D_{3}-9 C_{3}\left(D_{4}^{2}-D_{5} D_{3}\right)}{12 D_{3}^{3}} \ell^{4}+\frac{C_{4} D_{3}+3 C_{3} D_{4}}{12 D_{3}^{2}} \ell^{5}-\frac{5 C_{3}}{72 D_{3}} \ell^{6}\right) \Psi(h ; 0)
\end{aligned} .
$$

Although this example seems tedious and lengthy, it is worth mentioning that it yields some surprises. For instance, the careful reader will note that the $\operatorname{OPE} \Psi_{\left(h_{1} ; 1\right)}(z) \Psi_{\left(h_{2} ; 2\right)}(w)$ does not contain a term proportional to $\log \left(z_{12}\right)^{2}$. Of course, it is clear from general arguments that this particular OPE may contain terms proportional to $\log \left(z_{12}\right)^{k}$ for $k=$ $0,1,2,3$, where 3 is the total J-level involved. The fact that the square term is missing is due to the general structure of the OPEs as required by global conformal covariance.

It is illuminating to check the following: The three-point functions can be viewed as polynomials in the three variables $\ell=\log \left(z_{12}\right), \lambda=\log \left(z_{23}\right)$ and $\Lambda=\log \left(z_{13}\right)$. The twopoint functions can then be seen as polynomials in the one variable $\lambda=\log \left(z_{23}\right)$. Here, we again skip the trivial dependency on $\prod_{i<j} z_{i j}^{\mu_{i j}}$ factors. The interesting fact, which also provides an excellent consistency check, is that the matrix product of $G_{k_{1}, k_{2}, k}^{(3)}\left(z_{1}, z_{2}, z_{3}\right)$ with the inverse of the matrix $G_{k, k_{3}}^{(2)}\left(z_{2}, z_{3}\right)$ yields sums of products of polynomials, namely $\left(G_{k_{1}}^{(3)}[\ell, \lambda, \Lambda]\right)_{k_{2}, k}\left(G^{(2)}[\Lambda]\right)^{k, k_{3}}=\left(C_{k_{1}+k_{2}}^{k_{3}}[\ell, \lambda, \Lambda]\right)$, which always reduce for $\Lambda=\lambda$ to polynomials of the one variable $\ell$ only. To be more specific, the full set of two- and three-point functions as derived in this paper is indeed consistent with the above given OPE formula. In fact, since we have for the two-point functions

$$
G_{k_{2}, k_{3}}^{(2)}[\lambda]=\delta_{h_{2}, h_{3}}\left(\sum_{m=0}^{k_{2}+k_{3}} D_{\left(h_{2}, h_{3} ; k_{2}+k_{3}-m\right)} \frac{(-2)^{m}}{m !} \lambda^{m}\right) \mathrm{e}^{-\lambda\left(h_{2}+h_{3}\right)},
$$

and the three-point functions read

$$
G_{k_{1}, k_{2}, k_{3}}^{(3)}[\ell, \lambda, \Lambda]=\sum_{m=r-1}^{k_{1}+k_{2}+k_{3}} C_{\left(h_{1}, h_{2}, h_{3} ; m\right)} \sum_{j_{1}=0}^{k_{1}} \sum_{j_{2}=0}^{k_{2}} \sum_{j_{3}=0}^{k_{3}} \delta_{j_{1}+j_{2}+j_{3}, k_{1}+k_{2}+k_{3}-m}
$$




$$
\times \frac{1}{j_{1} ! j_{2} ! j_{3} !}\left(\partial_{h_{1}}\right)^{j_{1}}\left(\partial_{h_{2}}\right)^{j_{2}}\left(\partial_{h_{3}}\right)^{j_{3}}\left(\mathrm{e}^{\ell\left(h_{3}-h_{1}-h_{2}\right)} \mathrm{e}^{\Lambda\left(h_{2}-h_{1}-h_{3}\right)} \mathrm{e}^{\lambda\left(h_{1}-h_{2}-h_{3}\right)}\right)
$$

the matrix product for the computation of the OPE functions,

$$
\left.G_{k_{1}}^{(3)}[\ell, \lambda, \Lambda] \cdot\left(G^{(2)}[\lambda]\right)^{-1}\right|_{\Lambda=\lambda}=C_{k_{1}}[\ell] \mathrm{e}^{\ell\left(h_{3}-h_{1}-h_{2}\right)}
$$

yields a structure matrix with polynomial coefficients solely in the variable $\ell=\log \left(z_{12}\right)$.

In our example, we may for instance look at $\Psi_{\left(h_{1} ; 3\right)} \Psi_{\left(h_{2} ; 3\right)}$ and there at the factor in front of the leading term $\Psi_{(h ; 3)}$ on the right hand side. This factor results from the sum of appropriate products of the following expressions (notation as above):

$$
\begin{aligned}
&\left\langle\Psi_{\left(h_{1} ; 3\right)} \Psi_{\left(h_{2} ; 3\right)} \Psi_{(h ; 0)}\right\rangle=C_{6}-2 C_{5} \ell+2 C_{4} \ell^{2}-\frac{4}{3} C_{3} \ell^{3} \\
&\left\langle\Psi_{\left(h_{1} ; 3\right)} \Psi_{\left(h_{2} ; 3\right)} \Psi_{(h ; 1)}\right\rangle=C_{7}-C_{6}(\lambda+\Lambda)-\frac{1}{1} 2 C_{3}(\lambda-\Lambda)^{4}-\left(C_{6}-2 C_{5}(\lambda+\Lambda)\right) \ell \\
&-\left(\frac{1}{2} C_{3}(\lambda-\Lambda)^{2}+2 C_{4}(\lambda+\Lambda)\right) \ell^{2}+\frac{2}{3}\left(C_{4}+2 C_{3}(\lambda+\Lambda)\right) \ell^{3}-\frac{3}{4} C_{3} \ell^{4} \\
&\left\langle\Psi_{\left(h_{1} ; 3\right)} \Psi_{\left(h_{2} ; 3\right)} \Psi_{(h ; 2)}\right\rangle=C_{8}-C_{7}(\lambda+\Lambda)+\frac{1}{2} C_{6}(\lambda+\Lambda)^{2}-\frac{1}{1} 2 C_{4}(\lambda-\Lambda)^{4}+\frac{1}{1} 2 C_{3}(\lambda+\Lambda)(\lambda-\Lambda)^{4} \\
&-\left(C_{7}-C_{6}(\lambda+\Lambda)+C_{5}(\lambda+\Lambda)^{2}+\frac{1}{4} C_{3}(\lambda-\Lambda)^{4}\right) \ell \\
&+\frac{1}{2}\left(C_{6}+C_{4}\left(\lambda^{2}+\Lambda^{2}+6 \lambda \Lambda\right)+C_{3}(\lambda+\Lambda)(\lambda-\Lambda)^{2}\right) \ell^{2} \\
&-\frac{1}{3}\left(C_{5}+2 C_{4}(\lambda+\Lambda)+\frac{1}{2} C_{3}\left(5 \lambda^{2}+5 \Lambda^{2}+6 \lambda \Lambda\right)\right) \ell^{3} \\
&+\frac{1}{4}\left(C_{4}+3 C_{3}(\lambda+\Lambda)\right) \ell^{4}-\frac{1}{4} C_{3} \ell^{5} \\
&=C_{9}-C_{8}(\lambda+\Lambda)+\frac{1}{2} C_{7}(\lambda+\Lambda)^{2}-\frac{1}{6} C_{6}(\lambda+\Lambda)^{3}-\frac{1}{1} 2 C_{5}(\lambda-\Lambda)^{4} \\
&+\frac{1}{1} 2 C_{4}(\lambda+\Lambda)(\lambda-\Lambda)^{4}-\frac{1}{7} 2\left(5 \lambda^{2}+5 \Lambda^{2}+2 \lambda \Lambda\right)(\lambda+\Lambda)^{4} \\
&\left\langle\Psi_{\left(h_{1} ; 3\right)} \Psi_{\left(h_{2} ; 3\right)} \Psi_{(h ; 3)}\right\rangle \\
&-\left(C_{8}-C_{7}(\lambda+\Lambda)+\frac{1}{2} C_{6}(\lambda+\Lambda)^{2}-\frac{1}{3} C_{5}(\lambda+\Lambda)^{3}+\frac{1}{4} C_{4}(\lambda-\Lambda)^{4}-\frac{1}{4} C_{3}(\lambda+\Lambda)(\lambda-\Lambda)^{4}\right) \ell \\
&+\frac{1}{2}\left(C_{7}-C_{6}(\lambda+\Lambda)-C_{5}(\lambda-\Lambda)^{2}+\frac{1}{3} C_{4}\left(\lambda^{2}+\Lambda^{2}-10 \lambda \Lambda\right)(\lambda+\Lambda)\right. \\
&\left.-\frac{1}{4} C_{3}\left(3 \lambda^{2}+3 \Lambda^{2}+2 \lambda \Lambda\right)(\lambda-\Lambda)^{2}\right) \ell^{2} \\
&-\frac{1}{1} 8\left(3 C_{6}-6 C_{5}(\lambda+\Lambda)-3 C_{4}\left(\lambda^{2}+\Lambda^{2}+6 \lambda \Lambda-C_{3}\left(7 \lambda^{2}+7 \Lambda^{2}+2 \lambda \Lambda\right)(\lambda+\Lambda)\right) \ell^{3}\right. \\
&-\frac{1}{4}\left(\frac{1}{3} C_{5}+C_{4}(\lambda+\Lambda)\right) \ell^{4}+\frac{3}{2} C_{3}(\lambda+\Lambda)^{2}+\frac{1}{1} 2\left(C_{4}+3 C_{3}(\lambda+\Lambda)\right) \ell^{5}-\frac{5}{7} 2 C_{3} \ell^{6}
\end{aligned}
$$

for the three-point functions. Note that these expressions are all symmetric under the exchange $\lambda \leftrightarrow \Lambda$ as they should be. One needs also the two-point functions for which we have

$$
\begin{aligned}
& \left\langle\Psi_{(h ; 0)} \Psi_{(h ; 3)}\right\rangle=D_{3}, \\
& \left\langle\Psi_{(h ; 1)} \Psi_{(h ; 3)}\right\rangle=D_{4}-2 D_{3} \lambda, \\
& \left\langle\Psi_{(h ; 2)} \Psi_{(h ; 3)}\right\rangle=D_{5}-2 D_{4} \lambda+2 D_{3} \lambda^{2}, \\
& \left\langle\Psi_{(h ; 3)} \Psi_{(h ; 3)}\right\rangle=D_{6}-2 D_{5} \lambda+2 D_{4} \lambda^{2}-\frac{4}{3} D_{3} \lambda^{3} .
\end{aligned}
$$

\section{Non-Proper Primary Fields}

WE NOW TURN to the next complicated case, where we explicitly allow that the OPE of two primary fields might yield a logarithmic partner field on the right hand side. It is well known that such primary fields exist, in particular the so-called pre-logarithmic fields. In the $c=-2$ theory, the field $\mu$ with conformal weight $h=-1 / 8$ is such a field, since it is a 
Virasoro primary field with $\operatorname{OPE} \mu(z) \mu(w)=(z-w)^{1 / 4}(\tilde{\mathbb{I}}(w)-2 \log (z-w) \mathbb{I})$. However, the primary field $\mu$ is not itself part of a Jordan cell. The $c=-2$ theory provides another example of primary fields whose OPE also yields the field $\tilde{\mathbb{I}}$, namely the so-called fermionic fields $\theta_{\alpha}$. With our notation introduced in section II.1, the former pre-logarithmic fields are twist fields, i.e. fields with non-trivial boundary conditions. Such fields do not have a zero mode content. The latter fields, however, have a zero mode content with the property that $Z_{+}\left(\theta_{\alpha}\right) \neq Z_{-}\left(\theta_{\alpha}\right)$, since $Z_{\beta}\left(\theta_{\alpha}\right)=\delta_{\alpha \beta}$. These fields are again not members of Jordan cells.

Of course, one might imagine a situation where non-proper primary fields do form part of a Jordan cell. The problem then is, that it is no longer possible to solve the conformal Ward identities in a hierarchical manner, as in section III, without further knowledge about the operator algebra. Basically, our approach in the third section was to find the first non-vanishing two- and three-point functions, the ones with minimal zero mode content, and to derive correlators with higher zero-mode content by solving the inhomogeneous Ward identities step by step. If the zero mode content of all fields is known, we can estimate which two- and three-point functions might be non-zero, since the OPE must satisfy the bound

$$
Z_{0}\left(\Psi_{3}\right) \leq Z_{0}\left(\Psi_{1}\right)+Z_{0}\left(\Psi_{2}\right)
$$

for all fields $\Psi_{3}(w)$ in the operator product of $\Psi_{1}(z)$ with $\Psi_{2}(w)$. This would provide us with a starting point for the hierarchical solution scheme. Unfortunately, this does not work for the twist fields, because no zero mode content can be defined for them. There is so far no example known where twist fields form part of a Jordan cell. We don't see an easy way to extend our description of Jordan cells in terms of zero mode content of fields such that it would encompass twist fields within Jordan cells. Thus we leave investigation of this case for future work.

\section{V.1. FERMIONIC FIELDS}

Let us now concentrate on the best known case of a rank $r=2$ LCFT, i.e. where the maximal rank of Jordan cells is two, as in the prime example of the $c=-2$ theory. There, logarithmic operators, which together with their proper primary partners span the Jordan cells, are created by the operator $\tilde{\mathbb{I}}(z)=\Psi_{(0 ; 1)}(z)$. As long as no twist fields are considered, we can construct all fields in terms of the pair of anti-commuting scalar $\theta$ fields defined in section II.1. Remember that the $\xi$ modes become the creation operators for logarithmic states. It is easy to see that proper primary fields do not possess any of the $\xi$ zero modes, while logarithmic fields possess precisely the zero mode contribution $\frac{1}{2} \varepsilon^{\alpha \beta} \xi_{\alpha} \xi_{\beta}$. Since the $\xi$ modes do anti-commute, we call fields with just one $\xi$ zero mode fermionic, and fields which are quadratic in $\xi$ bosonic. This coincides with the fact that for $c=-2$ all logarithmic fields and all proper primary fields have integer conformal weights. However, nothing prohibits us from considering the fields $\theta_{\alpha}(z)$ themselves which also have zero conformal weight, but are fermionic. Many of the above arguments remain valid when we consider correlation functions involving $\theta$ fields. A further restriction is that the total number of $\theta$ fields must be even, since otherwise the correlation function vanishes identically. The reason is that consistency with the anti-commutation relations enforces to put $\left\langle\xi_{+}\right\rangle=\left\langle\xi_{-}\right\rangle=0$. Only when the total number of $\theta$ fields is even, do we have a chance that a term $\xi_{+} \xi_{-}$will survive after contraction. Moreover, the number of $\theta_{+}$and $\theta_{-}$fields must be equal, since otherwise $\theta_{ \pm, 0}$ zero modes will survive. 
More generally, a correlator of fields $\left\langle\Psi_{1}\left(z_{1}\right) \ldots \Psi_{n}\left(z_{n}\right)\right\rangle$ can only be non-zero, if it satisfies the conditions

$$
\sum_{i} Z_{+}\left(\Psi_{i}\right)=\sum_{i} Z_{-}\left(\Psi_{i}\right) \quad \text { and } \quad \sum_{i} Z_{0}\left(\Psi_{i}\right) \geq 2
$$

in the rank two case. This statement is valid for fields $\Psi_{i}$ which are either proper primaries, logarithmic partners or fermionic fields (i.e. fields with fermionic zero mode content).

Correlation functions involving fermionic fields can be computed along the same lines as set out above. The only difference is that the action of the Virasoro algebra on fermionic fields does not have an off-diagonal part in the rank two case. More precisely, this is true for the $L_{0}$ mode of the Virasoro algebra, since this mode reduces always the total zero mode content symmetrically, i.e. it reduces $Z_{-}$by the same amount as $Z_{+}$. Other modes, such as $L_{1}$, can reduce the zero mode content unevenly, as we will see in the next section. As long as we still assume that all fields in a Jordan cell are quasi-primary, we don't have to worry about this possibility. Of course, in LCFTs of higher rank, fermionic fields can easily admit an off-diagonal action of $L_{0}$, since $Z_{ \pm}$can both be larger than one. To keep the following formulæ reasonable simple, we won't consider this case here.

The important fact is that the OPE of two fermionic fields produces a logarithm, i.e.

$$
\theta_{\alpha}(z) \theta_{\beta}(0)=\varepsilon_{\alpha \beta}(\tilde{\mathbb{I}}(0)+(1+\log z) \mathbb{I}(0)) .
$$

This follows on general grounds, since $\left\langle\theta_{\alpha}(z) \theta_{\beta}(w)\right\rangle=\varepsilon_{\alpha \beta}$ such that a three-point function of two fermionic and one logarithmic field necessarily involves a logarithm. The argument remains valid in the general rank two case and fields of arbitrary scaling dimension. Each Jordan cell is extended by two fermionic sectors such that we have the four fields $\Psi_{(h ; 0)}$, $\Psi_{(h ; 1)}$, and $\Psi_{(h ; \pm)}$. It is then an easy task to compute all their OPEs from the two- and three-point functions

$$
\begin{aligned}
& \left\langle\Psi_{(h ;+)}\left(z_{1}\right) \Psi_{(h ;-)}\left(z_{2}\right)\right\rangle=\varepsilon_{+-} D_{(h, h ; \pm)}\left(z_{12}\right)^{-2 h}, \\
& \left\langle\Psi_{(h ; 0)}\left(z_{1}\right) \Psi_{(h ; 1)}\left(z_{2}\right)\right\rangle=D_{(h, h ; 1)}\left(z_{12}\right)^{-2 h}, \\
& \left\langle\Psi_{(h ; 1)}\left(z_{1}\right) \Psi_{(h ; 1)}\left(z_{2}\right)\right\rangle=\left(D_{(h, h ; 2)}-2 D_{(h, h ; 1)} \log z_{12}\right)\left(z_{12}\right)^{-2 h}, \\
& \left\langle\Psi_{\left(h_{1} ; 0\right)}\left(z_{1}\right) \Psi_{\left(h_{2} ; 0\right)}\left(z_{2}\right) \Psi_{\left(h_{3} ; 1\right)}\left(z_{3}\right)\right\rangle=C_{\left(h_{1}, h_{2}, h_{3} ; 1\right)}\left(z_{12}\right)^{h_{3}-h_{1}-h_{2}}\left(z_{13}\right)^{h_{2}-h_{1}-h_{3}}\left(z_{23}\right)^{h_{1}-h_{2}-h_{3}}, \\
& \left\langle\Psi_{\left(h_{1} ; 0\right)}\left(z_{1}\right) \Psi_{\left(h_{2} ;+\right)}\left(z_{2}\right) \Psi_{\left(h_{3} ;-\right)}\left(z_{3}\right)\right\rangle=\varepsilon_{+-} C_{\left(h_{1} ; 0\right)\left(h_{2}, h_{3} ; \pm\right)} \Pi\left(z_{i j}\right)^{h_{k}-h_{i}-h_{j}}, \\
& \left\langle\Psi_{\left(h_{1} ; 0\right)}\left(z_{1}\right) \Psi_{\left(h_{2} ; 1\right)}\left(z_{2}\right) \Psi_{\left(h_{3} ; 1\right)}\left(z_{3}\right)\right\rangle=\left(C_{\left(h_{1}, h_{2}, h_{3} ; 2\right)}-2 C_{\left(h_{1}, h_{2}, h_{3} ; 1\right)} \log z_{23}\right) \boldsymbol{\Pi}\left(z_{i j}\right)^{h_{k}-h_{i}-h_{j}}, \\
& \left\langle\Psi_{\left(h_{1} ; 1\right)}\left(z_{1}\right) \Psi_{\left(h_{2} ;+\right)}\left(z_{2}\right) \Psi_{\left(h_{3} ;-\right)}\left(z_{3}\right)\right\rangle=\varepsilon_{+-}\left(C_{\left(h_{1} ; 0\right)\left(h_{2}, h_{3} ; \pm\right)}\left(\log z_{23}-\log z_{12}-\log z_{13}\right)\right. \\
& \left.+C_{\left(h_{1} ; 1\right)\left(h_{2}, h_{3} ; \pm\right)}\right) \Pi\left(z_{i j}\right)^{h_{k}-h_{i}-h_{j}} \text {, } \\
& \left\langle\Psi_{\left(h_{1} ; 1\right)}\left(z_{1}\right) \Psi_{\left(h_{2} ; 1\right)}\left(z_{2}\right) \Psi_{\left(h_{3} ; 1\right)}\left(z_{3}\right)\right\rangle=\left(C_{\left(h_{1}, h_{2}, h_{3} ; 3\right)}-C_{\left(h_{1}, h_{2}, h_{3} ; 2\right)}\left(\log z_{12}+\log z_{13}+\log z_{23}\right)\right. \\
& +2 C_{\left(h_{1}, h_{2}, h_{3} ; 1\right)}\left(\log z_{12} \log z_{13}+\log z_{12} \log z_{23}+\log z_{13} \log z_{23}\right. \\
& \left.\left.-\frac{1}{2} \log ^{2} z_{12}-\frac{1}{2} \log ^{2} z_{13}-\frac{1}{2} \log ^{2} z_{23}\right)\right) \Pi\left(z_{i j}\right)^{h_{k}-h_{i}-h_{j}},
\end{aligned}
$$

and permutations. Note that we have explicitly indicated the antisymmetry under exchanging the order of the fermionic fields. These results agree in the special case where all $h_{i}=0$ with the explicit calculations for the $c=-2$ LCFT by Kausch [34]. The singular terms of the corresponding additional OPEs read

$$
\Psi_{\left(h_{1} ; 0\right)}\left(z_{1}\right) \Psi_{\left(h_{2} ; \pm\right)}\left(z_{2}\right)=\sum_{h} \frac{C_{\left(h_{1} ; 0\right)\left(h_{2}, h ; \pm\right)}}{D_{\left(h_{2}, h ; \pm\right)}}\left(z_{12}\right)^{h-h_{1}-h_{2}} \Psi_{(h ; \pm)}\left(z_{2}\right),
$$




$$
\begin{gathered}
\Psi_{\left(h_{1} ; \pm\right)}\left(z_{1}\right) \Psi_{\left(h_{2} ; \mp\right)}\left(z_{2}\right)=\sum_{h} \varepsilon_{\mp \pm}\left[\left(\frac{C_{\left(h_{1}, h_{2} ; \pm\right)(h ; 0)} D_{\left(h_{2}, h ; 2\right)}-C_{\left(h_{1}, h_{2} ; \pm\right)(h ; 1)} D_{\left(h_{2}, h ; 1\right)}}{D_{\left(h_{2}, h ; 1\right)}^{2}}\right.\right. \\
\left.\left.-\frac{C_{\left(h_{1}, h_{2} ; \pm\right)(h ; 0)}}{D_{\left(h_{2}, h ; 1\right)}} \log \left(z_{12}\right)\right) \Psi_{(h ; 0)}\left(z_{2}\right)+\frac{C_{\left(h_{1}, h_{2} ; \pm\right)(h ; 0)}}{D_{\left(h_{2}, h ; 1\right)}} \Psi_{(h ; 1)}\left(z_{2}\right)\right]\left(z_{12}\right)^{h-h_{1}-h_{2}}, \\
\Psi_{\left(h_{1} ; 1\right)}\left(z_{1}\right) \Psi_{\left(h_{2} ; \pm\right)}\left(z_{2}\right)=\sum_{h} \frac{C_{\left(h_{1} ; 1\right)\left(h_{2}, h ; \pm\right)}-C_{\left(h_{1} ; 0\right)\left(h_{2}, h, \pm\right)} \log \left(z_{12}\right)}{D_{\left(h_{2}, h ; \pm\right)}}\left(z_{12}\right)^{h-h_{1}-h_{2}} \Psi_{(h ; \pm)}\left(z_{2}\right) .
\end{gathered}
$$

The above statement shows that rank two LCFTs naturally allow for fermionic fields. It has been suggested in [61] to formally collect these quadruples of fields in "superfields" $\Psi_{h}\left(z, \eta^{+}, \eta^{-}\right)$of $N=2$ Grassmann variables such that

$$
\Psi_{h}\left(z, \eta^{+}, \eta^{-}\right)=\Psi_{(h ; 0)}(z)+\eta^{+} \Psi_{(h ;-)}(z)+\eta^{-} \Psi_{(h ;+)}(z)+\eta^{+} \eta^{-} \Psi_{(h ; 1)}(z),
$$

which in the $c=-2$ case resembles the $\xi_{ \pm}$zero mode contributions. It is tempting to conjecture that a rank $k$ LCFT will naturally incorporate the analog of anti-commuting scalars for $\mathbb{Z}_{k}$ para-fermions, whose OPEs among them create logarithmic fields of according J-levels. However, an investigation of this is beyond the scope of the present paper.

\section{V.2. Twist FiELDS}

As already explained, there is (at least) one more sort of fields which may occur in LCFTs. In the standard $c=-2$ example, the two fields $\mu(z)$ and $\sigma(z)$ with conformal weights $h_{\mu}=-1 / 8$ and $h_{\sigma}=3 / 8$ respectively, are not yet accounted for. These fields are twist fields. They can be treated much along the same lines as fermionic fields. The difference is that their mode expansion is in $\mathbb{Z}+\iota$ with a certain rational $\iota$ depending on the boundary conditions and the ramification number of the twists. The fields $\mu$ and $\sigma$ are $\mathbb{Z}_{2}$ twists. Despite the difference in the mode expansion, twist fields behave quite similar to the (para-)fermionic fields mentioned above. In particular, their two-point functions are nonzero if and only if they involve a twist $\chi_{\iota}$ and its anti-twist $\chi_{\iota^{*}}$, which resembles the fact that for fermionic fields only the two-point function of two different fermions is non-zero. Higher twist fields are then analogous to para-fermions.

One might attempt to extend the definition of zero mode content to rational numbers such that a twist field with mode expansion in $\mathbb{Z}+\iota$ would get assigned $Z_{0}\left(\chi_{\iota}\right)=Z_{+}\left(\chi_{\iota}\right)+$ $Z_{-}\left(\chi_{\iota}\right)=\iota+\iota^{*}$. A necessary condition for a correlator $\left\langle\chi_{\iota_{1}} \ldots \chi_{\iota_{n}}\right\rangle$ to be non-zero would then read

$$
\sum_{i} Z_{+}\left(\chi_{\iota_{i}}\right) \in \mathbb{Z} \quad \text { and } \quad \sum_{i} Z_{-}\left(\chi_{\iota_{i}}\right) \in \mathbb{Z}
$$

However, such an assignment is obviously only determined modulo integers, and it is not a priory clear how to implement the condition for a minimal zero mode content in general. At least, one should now always consider separately the zero mode content $Z_{+}$and $Z_{-}$, as indicated above. In the rank two case, however, we can incorporate the condition for a minimal zero mode content into (5.16) by simply replacing $\mathbb{Z}$ by $\mathbb{N}$. Indeed, the $\mathbb{Z}_{2}$ twist fields $\mu$ and $\sigma$ have twist numbers $\left(\iota, \iota^{*}\right)=\left(\frac{1}{2}, \frac{1}{2}\right)$ and $\left(-\frac{1}{2}, \frac{3}{2}\right)$ respectively, from which we immediately can read off, which two- and three-point functions of $\mu$ and $\sigma$ fields can be non-zero.

To emphasize the common features of fermionic and twist fields, we contrast their possible two- and three-point functions with the ones for fermionic fields (there are no 
non-vanishing two- or three-point functions involving both, fermionic and twist fields, simultaneously). The notation $\iota^{*}$ means the anti-twist $1-\iota$ with respect to $\iota$, and one always has $h_{\iota}=h_{\iota^{*}}$. The only nontrivial two-point function then reads

$$
\left\langle\chi_{\iota}\left(z_{1}\right) \chi_{\iota^{*}}\left(z_{2}\right)\right\rangle=D_{\iota \iota^{*}}\left(z_{12}\right)^{-2 h_{\iota}}
$$

with $D_{\iota \iota^{*}}=D_{\iota^{*} \iota}$. Note that in contrast to the fermionic fields, twist fields are symmetric. The three-point functions are easily computed and the results are

$$
\begin{aligned}
\left\langle\Psi_{\left(h_{1} ; 0\right)} \Psi_{\left(h_{2} ; k^{\prime}\right)} \chi_{\iota_{3}}\right\rangle & =0, \\
\left\langle\Psi_{\left(h_{1} ; 0\right)} \chi_{\iota_{2}} \chi_{\iota_{3}}\right\rangle & =\delta_{\iota_{3}, \iota_{2}^{*}} C_{\left(h_{1}, 0\right) \iota_{2} \iota_{3}^{*}} \Pi\left(z_{i j}\right)^{h_{k}-h_{i}-h_{j}}, \\
\left\langle\Psi_{\left(h_{1} ; 1\right)} \chi_{\iota_{2}} \chi_{\iota_{3}}\right\rangle & =\delta_{\iota_{3}, \iota_{2}^{*}}\left(C_{\left(h_{1}, 1\right) \iota_{2} \iota_{3}^{*}}+C_{\left(h_{1}, 0\right) \iota_{2} \iota_{3}^{*}}\left(\log z_{23}-\log z_{12}-\log z_{13}\right)\right) \Pi\left(z_{i j}\right)^{h_{k}-h_{i}-h_{j}}, \\
\left\langle\chi_{\iota_{1}} \chi_{\iota_{2}} \chi_{\iota_{3}}\right\rangle & =\left(\delta_{\iota_{3}, \iota_{1}^{*}+\iota_{2}^{*}}+\delta_{\iota_{3}, 1-\iota_{1}-\iota_{2}}\right) C_{\iota_{1} \iota_{2} \iota_{3}} \Pi\left(z_{i j}\right)^{h_{k}-h_{i}-h_{j}}, \\
\left\langle\Psi_{\left(h_{1} ; 1\right)} \Psi_{\left(h_{2} ; 1\right)} \chi_{\iota_{3}}\right\rangle & =C_{\left(h_{1}, h_{2} ; 2\right) \iota_{3}} \Pi\left(z_{i j}\right)^{h_{k}-h_{i}-h_{j}} .
\end{aligned}
$$

Note that some of the introduced constants may be zero, e.g. $C_{\iota_{1} \iota_{2} \iota_{3}}=0$ whenever the three twists do not add up to an integer. Most remarkably is perhaps the fact that $\left\langle\Psi_{\left(h_{1} ; 1\right)} \Psi_{\left(h_{2} ; 1\right)} \chi_{\iota 3}\right\rangle$ might be non-zero. This does not happen in the $c=-2$ theory, since it implies that the OPE of two logarithmic fields has a contribution

$$
\Psi_{(h ; 1)}(z) \Psi_{\left(h^{\prime} ; 1\right)}(0)=\ldots+\frac{C_{\left(h, h^{\prime} ; 2\right) \iota}}{D_{\iota \iota^{*}}} z^{h_{\iota}-h-h^{\prime}} \chi_{\iota}(0)+\ldots
$$

which is not the case in the $c=-2$ theory. However, already the next theory in the $c_{p, 1}$ series of LCFTs, namely the $c_{3,1}=-7$ model, shows precisely this feature, where the fusion rule of the $h=0$ logarithmic field with itself involves the twist field with $h=-\frac{1}{3}$ on the right hand side. Since the main focus of this paper lies on logarithmic fields, we will not go into further detail here. The OPEs involving $\chi_{\iota}$ fields read correspondingly

$$
\begin{aligned}
\Psi_{(h ; 0)}(z) \chi_{\iota}(0) & =\frac{C_{(h ; 0) \iota \iota^{\prime}}}{D_{\iota \iota^{*}}} z^{h_{\iota^{\prime}}-h_{\iota}-h} \chi_{\iota^{\prime}}(0), \\
\Psi_{(h ; 1)}(z) \chi_{\iota}(0) & =\frac{C_{\left(h, h^{\prime} ; 2\right) \iota}}{D_{(h ; 1)}} z^{h^{\prime}-h-h_{\iota}} \Psi_{\left(h^{\prime} ; 0\right)} \\
& +\frac{C_{(h ; 1) \iota \iota^{\prime}}-C_{(h ; 0) \iota \iota^{\prime}} \log z}{D_{\iota \iota^{*}}} z^{h_{\iota^{\prime}}-h_{\iota}-h} \chi_{\iota^{\prime}}(0)+\frac{C_{(h ; 1) \iota \iota^{\prime *}}}{D_{\iota \iota^{*}}} z^{h_{\iota^{\prime *}}-h_{\iota}-h} \chi_{\iota^{\prime *}}(0), \\
\chi_{\iota}(z) \chi_{\iota^{\prime}}(0) & =\frac{C_{(h ; 1) \iota \iota^{\prime}}}{D_{(h ; 1)}} z^{h^{\prime}-h_{\iota}-h_{\iota^{\prime}}} \Psi_{(h ; 0)}(0)+\frac{C_{\iota \iota^{\prime} \iota^{\prime \prime}}}{D_{\iota \iota^{*}}} z^{h_{\iota^{\prime \prime}}-h_{\iota}-h_{\iota^{\prime}}} \chi_{\iota^{\prime \prime}}(0), \\
\chi_{\iota}(z) \chi_{\iota^{*}}(0) & =\frac{C_{(h ; 1) \iota^{*}} D_{(h ; 1)}-C_{(h ; 0) \iota \iota^{*}} D_{(h ; 2)}+C_{(h ; 0) \iota \iota^{*}} D_{(h ; 1)} \log z}{D_{(h ; 1)}^{2}} z^{h-h_{\iota}-h_{\iota^{*}}} \Psi_{(h ; 0)}(0) \\
& +\frac{C_{(h ; 0) \iota \iota^{*}}}{D_{(h ; 1)}} z^{h-h_{\iota}-h_{\iota^{*}}} \Psi_{(h ; 1)}(0),
\end{aligned}
$$

where in the last two equations $\iota^{\prime} \neq \iota^{*}$. As remarked above, some of the structure constants may vanish, as they do in the $c=-2$ LCFT. One sees that even the simple rank two case gets quite complicated and needs a cumbersome notation. The situation is slightly better in the particular case for the $c=-2$ theory where all amplitudes involving up to four twist fields as well as amplitudes with an arbitrary number of fermionic fields were computed in 34 . 


\section{NON-QUASI-PRIMARY FIELDS}

OUR DISCUSSION OF correlation functions and operator product expansions in logarithmic CFTs heavily relies on the following assumption which we so far have made: that all logarithmic partner fields within a Jordan cell be quasi-primary. This means in particular that $L_{1}|h ; k\rangle=L_{1} \Phi_{(h ; k)}(0)|0\rangle=0$. As a consequence, we could make elaborate use of the Ward identities of global conformal transformations in the form (3.2). This section is devoted to the question under which more relaxed circumstances our results still hold.

It is by no means clear that logarithmic partner fields are indeed all quasi-primary. On the contrary, even the simplest known LCFT, the $c=-2$ model, features a Jordan cell where the logarithmic partner is not quasi-primary [21]. Actually, as already outlined in section II, in this model exists a Jordan cell for conformal weight $h=1$, built from a primary field $\partial \theta_{\alpha}(z)$ and its logarithmic partner field $: \theta_{+} \theta_{-} \partial \theta_{\alpha}:(z)$. Here, we again used the realization of the $c=-2$ theory in terms of two anti-commuting scalar fields $\theta_{ \pm}(z)$ along the lines (2.10) and (2.11). It is easy to see that $|1 ; 0\rangle=\theta_{\alpha,-1}|0\rangle$ and that $|1 ; 1\rangle=\theta_{\alpha,-1}\left(\xi_{+} \xi_{-}+1\right)|0\rangle$. It follows that $L_{1}|1 ; 0\rangle=0$, while $L_{1}|1 ; 1\rangle=-\xi_{\alpha}|0\rangle \neq 0$, where one uses that the stress-energy tensor is given by (2.9). We remind the reader that $\xi_{\alpha}$ is one of the two zero-modes of the field $\theta_{\alpha}(z)$. Hence, $|1 ; 1\rangle$ is not a state corresponding to a quasi-primary field.

The point is that it does not matter. The global Ward identities are not affected by this non-zero term. More generally, all correlation functions involving the field $\Psi_{(h=1 ; 1)}(z)$, the logarithmic partner of the primary $h=1$ field $\Psi_{(h=1 ; 0)}(z)=\partial \theta(z)$, behave exactly as if the field were quasi-primary. The reason for this is simply that the state $L_{1}|1 ; 1\rangle$ is fermionic with respect to the number of $\xi_{\alpha}$ zero modes. More precisely, it has $Z_{+}=1$, $Z_{-}=0$ (or vice versa). Any correlation function can only be non-zero if the numbers $\left(Z_{+}, Z_{-}\right)$of $\xi_{ \pm}$zero modes are, after all contractions are done, exactly $(1,1)$. Hence, any correlation function involving $\Psi_{(h=1 ; 1)}(z)$, which has a chance to be non-zero, must initially have fermion numbers $\left(Z_{+}, Z_{-}\right)$with $Z_{+}+Z_{-}$even and $Z_{+} \geq 1, Z_{-} \geq 1$. Applying $L_{1}$ to it results in the global conformal Ward identity up to additional terms with fermion numbers $\left(Z_{+}-1, Z_{-}\right)$or $\left(Z_{+}, Z_{-}-1\right)$. Since $Z_{+}+Z_{-}-1$ is then necessarily an odd number, the additional terms must vanish. It follows that the fact that $\Psi_{(h=1 ; 1)}(z)$ is not quasi-primary does not influence the correlation functions, because the spoiling term does not lead to any non-vanishing contributions. Note that this statement is only true as long as we consider the effect within correlation functions. The deeper reason is that the action of the Virasoro algebra changes the fermion numbers unevenly in this case.

Of course, not all correlation functions involving a field $\theta_{\alpha}(z)$ corresponding to the state $\xi_{\alpha}|0\rangle$ automatically vanish. For example, $\left\langle 0\left|\theta_{+}(z) \theta_{-}(w)\right| 0\right\rangle \neq 0$. What is meant in the above discussion is that all correlation functions vanish, which result from applying $L_{1}$ to a correlator involving $\Psi_{(h=1 ; 1)}(z)$ and other fields such that the initial correlator might be non-zero. Since $L_{1}$ acts as a derivation, it only changes one of the inserted fields at a time, so that starting with an admissible number of $\xi_{\alpha}$ zero modes leads to terms with non-admissible numbers of $\xi_{\alpha}$ zero modes.

There are strong indications that this structure of Jordan cells with non-quasi-primary fields is more generally true. At least, there is so far no LCFT explicitly known where logarithmic partner fields are not quasi-primary in a way which would affect correlation functions and therefore our general conclusions on their general structure. All LCFTs

\footnotetext{
$\dagger$ More precisely, we are dealing with a doublet of two such fields, distinguished by the $\alpha$-label.
} 
which can be constructed or realized explicitly in terms of fundamental free fields, such as the $c=-2$ model, receive their peculiar logarithmic fields ultimately due to the existence of conjugate pairs of zero-modes. In the $c=-2$ model, these are the two pairs $\xi_{ \pm}, \theta_{\mp, 0}$ respectively (see sect. II.1). In this situation, we only need that the logarithmic partner fields are quasi-primary up to terms with the "wrong" number of such zero modes. Here, wrong means in the above explained sense that the number of zero modes gets changed unevenly.

Following the lines of [61, the effective zero mode content is equivalently described in terms of nilpotent variables with which the fields spanning a Jordan cell are grouped together in a superfield like fashion. In the rank two case this is easily accomplished by introducing for each Jordan cell two Grassmann variables $\eta^{ \pm}$and a bosonic nilpotent variable $\Theta=\eta^{+} \eta^{-}$with the property $\Theta^{2}=0$. Then, we may group together all fields, the primary $\Psi_{(h ; 0)}(z)$, its logarithmic partner $\Psi_{(h ; 1)}(z)$ and the two fermionic fields $\Psi_{(h ; \pm)}(z)$ as $\Psi_{h}(z)=\Psi_{(h ; 0)}(z)+\eta^{+} \Psi_{(h ;+)}(z)+\eta^{-} \Psi_{(h ;-)}(z)+\eta^{+} \eta^{-} \Psi_{(h ; 1)}(z)$. For higher rank Jordan cells, an analogous procedure applies. For a Jordan cell of even rank $2 r$ one needs $r-1$ pairs of Grassmann variables. The member of J-level $k$ in the Jordan cell itself is given by the elementary completely symmetric polynomial $\sigma_{k}\left(\Theta_{1}, \ldots, \Theta_{r-1}\right)=\sigma_{k}\left(\eta_{1}^{+} \eta_{1}^{-}, \ldots, \eta_{r-1}^{+} \eta_{r-1}^{-}\right)$ of total degree $2 k$ in the $\eta$ 's. So the primary field $\Phi_{(h ; 0)}(z)$ belongs to $\sigma_{0} \equiv 1$, while the top J-level field $\Phi_{(h ; r-1)}(z)$ belongs to $\sigma_{r-1} \propto \eta_{1}^{+} \eta_{1}^{+} \ldots \eta_{r-1}^{+} \eta_{r-1}^{-}$. The elementary completely symmetric polynomial $\sigma_{k}\left(x_{1}, \ldots, x_{n}\right)$ is defined as $\sigma_{k}=\sum_{i_{1}<i_{2}<\ldots i_{k}} x_{i_{1}} x_{i_{2}} \ldots x_{i_{k}}$ up to normalization. Other polynomials $p\left(\left\{\eta_{i}^{ \pm}\right\}\right)$, whose monomials $m_{\{i\}}=\prod_{k} \eta_{i_{k}}^{\alpha_{k}}$ have differing partial degrees $\operatorname{deg}_{\eta_{j}^{+}}\left(m_{\{i\}}\right) \neq \operatorname{deg}_{\eta_{j}^{-}}\left(m_{\{i\}}\right)$ for at least one $j$, belong to fields which are the higher rank analogons of the fermionic fields described above. Overall symmetry in the Grassmann variables demands that all polynomials must be symmetric polynomials in the $\eta$ 's. However, what may be varied is how the total degree is split into $\eta^{+}$variables and $\eta^{-}$variables. Hence, we use the symmetric polynomials in two colors, $\sigma_{k}^{l, k-l}$, instead. These can be written as $\sigma_{k}^{l, k-l}\left(\left\{\eta^{+}, \eta^{-}\right\}\right)=\sigma_{\max (l, k-l)}(\{\Theta\}) \sigma_{\min (l, k-l)}\left(\left\{\eta_{\alpha_{\min (l, k-l)}}\right\}\right)$. Note that $\sigma_{k}^{l, k-l}=0$ for $l$ or $k-l$ larger $r-1$. The Jordan cell is then spanned by the fields corresponding to the symmetric polynomials $\sigma_{2 k}^{k, k}\left(\left\{\eta^{+}, \eta^{+}\right\}\right)=\sigma_{k}(\{\Theta\})$.

For example, in the rank four case we have the following possibilities: The Jordan cell is spanned by

$$
\begin{aligned}
\boldsymbol{\Phi}_{h}(z)= & \Phi_{(h ; 0)}(z)+\sum_{1 \leq i \leq 3} \eta_{i}^{+} \eta_{i}^{-} \Phi_{(h ; 1)}(z)+\sum_{1 \leq i<j \leq 3} \eta_{i}^{+} \eta_{i}^{-} \eta_{j}^{+} \eta_{j}^{-} \Phi_{(h ; 2)}(z) \\
& +\eta_{1}^{+} \eta_{1}^{-} \eta_{2}^{+} \eta_{2}^{-} \eta_{3}^{+} \eta_{3}^{-} \Phi_{(h ; 3)}(z) \\
= & \sigma_{0} \Phi_{(h ; 0)}(z)+\sigma_{2}^{1,1} \Phi_{(h ; 1)}(z)+\sigma_{4}^{2,2} \Phi_{(h ; 2)}(z)+\sigma_{6}^{3,3} \Phi_{(h ; 3)}(z) .
\end{aligned}
$$

Furthermore, we have symmetrized non-bosonic fields according to the following "diamond" of two-color symmetric polynomials:

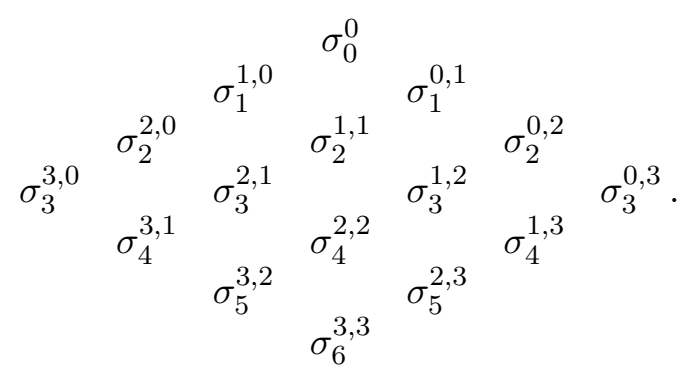


Of course, it is not clear whether all higher rank LCFTs fall into this pattern, but the crucial role of conjugate zero mode pairs suggests so.

It is now easy to see, which correlation functions may be non-zero. Clearly, each inserted field $\Phi$ comes with an associated polynomial $\sigma$. Consequently, an arbitrary $n$ point function can only be non-vanishing, if the product $\prod_{i=1}^{n} \sigma_{k_{i}}^{l_{i}, k_{i}-l_{i}}$ meets the conditions

$$
\sum_{i=1}^{n} l_{i}=\sum_{i=1}^{n}\left(k_{i}-l_{i}\right) \geq r-1 .
$$

Of course, this is nothing else than our initial conditions on the zero mode content, since any field with associated polynomial $\sigma_{k}^{l, k-l}$ has zero mode content $Z_{+}=l, Z_{-}=k-l$.

The action of symmetry generators such as the modes of the Virasoro algebra may have off-diagonal contributions meaning that they change a field into another and thus change a given associated polynomial into another. However, this off-diagonal term contributes to the correlator only if the resulting product of associated polynomials again satisfies the above condition (6.1). Now, symmetry generators always act as derivations on correlators such that an immediate necessary condition on an off-diagonal term is that the off-diagonal action of the generator moves vertically in the $\sigma$-diamond. Otherwise, the off-diagonal term has no effect in the correlator. Coming back to our initial example in the $c=-2$ theory, we have that $|1 ; 1\rangle$ has associated polynomial $\sigma_{2}^{1,1}$, while $L_{1}|1 ; 1\rangle$ has associated polynomial $\sigma_{1}^{1,0}$. Thus, $L_{1}$ does not move vertically in the $\sigma$-diamond, and the non-quasiprimary term hence does not contribute.

We therefore arrive at the following generalized picture: The action of symmetry generators such as the Virasoro algebra in a logarithmic CFT possesses off-diagonal additional terms with accompanying moves in the associated $\sigma$-diamond. However, all such terms with a non-vertical move are irrelevant when considered within correlation functions. All moves in the $\sigma$-diamond are obviously generated by the two basic moves

$$
Q^{+}: \sigma_{k}^{l, k-l} \mapsto \sigma_{k-1}^{l, k-1-l} \quad \text { and } \quad Q^{-}: \sigma_{k}^{l, k-l} \mapsto \sigma_{k-1}^{l-1, k-l}
$$

The careful reader might notice that these moves always go upwards within the diamond. However, since symmetry generators are presumably free of zero modes which do not annihilate the vacuum (otherwise, the vacuum is no longer invariant under the considered symmetry), we do not expect that a symmetry generator will move downwards within the diamond. These two basic moves may be viewed as generating a BRST like symmetry, since with $\left\langle\prod_{i} \sigma_{k_{i}}^{l_{i}, k_{i}-l_{i}}\right\rangle \neq 0$, we certainly have

$$
Q^{+}\left\langle\prod_{i} \sigma_{k_{i}}^{l_{i}, k_{i}-l_{i}}\right\rangle=\sum_{j}\left\langle\sigma_{k_{1}}^{l_{1}, k_{1}-l_{1}} \ldots \sigma_{k_{j}-1}^{l_{j}, k_{j}-1-l_{j}} \ldots \sigma_{k_{n}}^{l_{n}, k_{n}-l_{n}}\right\rangle=0
$$

and analogously for $Q^{-}$. Actually, one may introduce operators $Q_{\ell}^{ \pm}$with $1 \leq \ell \leq r-1$ which act by setting $\eta_{\ell}^{\mp}$ formally to one by contracting with a conjugate Grassmann variable, i.e. by acting with $\left\{\bar{\eta}_{\ell}^{\mp}, \cdot\right\}$. These latter operators have the nice property to automatically satisfy $\left(Q_{\ell}^{ \pm}\right)^{2}=0$, while $Q_{\ell}^{+} Q_{\ell}^{-} \neq 0$. These latter operators may then indeed be considered as BRST symmetries on correlation functions. We introduce $Q_{ \pm}$as symbols for classes of such symmetries, denoting arbitrary moves upwards in the diamond, which are not vertical. Thus, these classes contain also products such as $Q_{\ell_{1}}^{+} Q_{\ell_{2}}^{+}$etc.

We may thus finally generalize our assumptions on the fields in LCFTs such that the action of the Virasoro algebra only needs to satisfy our basic conditions (3.1) and (3.2) up 
to terms, which can be written as $Q_{ \pm}$of an admissible configuration, i.e. can be written as $Q_{ \pm}\left\langle\prod_{i} \sigma_{k_{i}}^{l_{i}, k_{i}-l_{i}}\right\rangle$ with $\left\langle\prod_{i} \sigma_{k_{i}}^{l_{i}, k_{i}-l_{i}}\right\rangle$ satisfying (6.1). If this is the case, all our results on two- and three-point functions and on the OPE structure remain valid in precisely the form as we have given them. All explicitly known LCFTs seem to possess only Jordan cells (and associated non-bosonic fields) which fall into this pattern. However, we do not have a proof that this has always to be the case. The strongest indications in favor of such a conjecture stem from the classification of possible Jordan structure like modules in the representation theory of the Virasoro algebra as given in [72]. A deeper study, which also should consider Jordan cell structures with respect to other chiral symmetries has to be left for future work.

Last but not least, we remark that the zero mode content further implies (2.17) and hence $(2.16)$. Let us momentarily assume that these bounds would not be strict. Then, for example, a two-point function $\left\langle\Psi_{(h ; 0)}(z) \Psi_{(h ; 0)}(w)\right\rangle$ could be non-zero, if the OPE of the primary field $\Psi_{(h ; 0)}$ with itself contained $\Psi_{(0 ; r-1)}$ in the rank $r$ case. It is an easy task to check that this is, however, inconsistent, since no solution for, say, $\left\langle\Psi_{(h ; 1)}(z) \Psi_{(h ; 0)}(w)\right\rangle$ can be found that satisfies all three Ward identities (3.2). On the other hand, two-point functions are insensitive to non-quasi-primary contributions, since these always would result in a two-point function of fields of differing conformal weights. Similar inconsistencies appear when other cases violating (2.16) are considered.

The higher rank case is more complicated, since OPEs might yield terms of too high J-level $k$, which do not influence the two-point functions as long as $k<r-1$. One has to consider three-point functions: Assume that e.g. the OPE $\Psi_{\left(h_{1} ; 0\right)} \Psi_{\left(h_{2} ; 0\right)}$ produces a term of maximal J-level $k$ with conformal weight $h_{3}$. Consider $\left\langle\Psi_{\left(h_{1} ; 0\right)} \Psi_{\left(h_{2}, 0\right)} \Psi_{\left(h_{3}, l\right)}\right\rangle$ with $l$ being the minimal J-level such that this three-point function is non-zero, and thus has the canonical form $C_{\left(h_{1} ; 0\right)\left(h_{2} ; 0\right)\left(h_{3} ; l\right)} \Pi\left(z_{i j}\right)^{h_{k}-h_{i}-h_{j}}$. Then, one easily finds solutions for $\left\langle\Psi_{\left(h_{1} ; 0\right)} \Psi_{\left(h_{2}, 0\right)} \Psi_{\left(h_{3}, l+1\right)}\right\rangle$ and $\left\langle\Psi_{\left(h_{1} ; 0\right)} \Psi_{\left(h_{2}, 1\right)} \Psi_{\left(h_{3}, l\right)}\right\rangle$. With these solutions as inhomogeneities in the Ward identities (3.2), no consistent solution for $\left\langle\Psi_{\left(h_{1} ; 0\right)} \Psi_{\left(h_{2}, 1\right)} \Psi_{\left(h_{3}, l+1\right)}\right\rangle$ can be found. Thus, we believe that LCFTs without a gradation in the J-level are not consistent, although we do not have a complete proof for this conjecture.

\section{CONCLUSION}

TAKING INTO ACCOUNT the proper action of the Virasoro algebra on logarithmic fields, i.e. working with Jordan cell representations as generalizations of irreducible highestweight representations [72], allows to evaluate OPEs in LCFT in a similar fashion as in ordinary CFT. The main difference is that each $n$-point function represents a full hierarchy of conformal blocks involving $s=1, \ldots, n$ logarithmic fields with varying J-levels $k_{i}=0, \ldots r-1$. While in ordinary conformal field theory it suffices to know correlation functions of primary fields only (since everything else is fixed by conformal covariance), in logarithmic CFT one needs to know the full hierarchy of $r^{n}$ correlations functions of primaries and all their logarithmic partner fields. Actually, there are only $r^{n}-\left(\begin{array}{c}n+r-2 \\ r-2\end{array}\right)$ different such correlation functions, since the total J-level must be at least $r-1$. If one additionally takes into account, that all correlation functions with total J-level precisely equal to the minimal value $r-1$ are identical (and do not involve any logarithms), the total number of different correlation functions reduces finally to $r^{n}-\left(\begin{array}{c}n+r-1 \\ r-1\end{array}\right)+1$.

The solution of this hierarchy can be obtained step by step, where the case with one logarithmic field only of maximal J-level is worked out in the same way as in ordinary CFT. The same holds for correlation functions with several logarithmic fields, such that 
the total J-level adds up to $r-1$. In each further step, the differential equations, which result from the existence of null vectors, are inhomogeneous, with the inhomogeneity determined by the conformal blocks of correlators with fewer logarithmic fields. Details on the computation of four-point functions have been presented in the last reference in [16]. Of course, since the OPEs are entirely determined in terms of the two- and three-point functions, the situation is simpler. As we have shown, these are - completely analogous to the case of ordinary CFT - already fixed up to constants by global conformal covariance. The formulae (3.9) and (3.11) yield the full hierarchy of these functions in a direct way.

With the help of a full set of two- and three-point functions, OPEs are then simply obtained through certain matrix products. Their non-trivial structure is essentially due to the fact that the matrix of two-point functions must be inverted first. The long example given in section IV demonstrates that this inversion results in many additional terms and non-trivial linear combinations of the involved structure constants which were not accounted for in older approaches, e.g. the one taken in [61]. In particular, it is not yet clear, how the otherwise elegant method of [61] to write correlation functions as formal power series expansions in nilpotent conformal weights $h+\eta, \eta^{r}=0$, can be transferred to operator product expansions. Nonetheless, we believe that our discussion on the structure matrix coefficients in terms of polynomials might be of help here.

This fills one of the few remaining gaps to put LCFT on equal footing with better known ordinary CFTs such as minimal models. The success of conformal field theory is mainly rooted in the fact that correlation functions can be computed effectively and exactly. The basic tools to achieve this are operator product expansions and differential equations due to the existence of null-vectors. Now, all these tools are also available in the logarithmic case. It is worth mentioning the following difference between the ordinary and the logarithmic case: In the ordinary case, any OPE $\Phi_{h_{i}} \Phi_{h_{j}}$ one wishes to compute depends only on two constants per primary field $\Phi_{h_{k}}$ occurring on the right hand side, namely $C_{h_{i} h_{j} h_{k}}$ and $D^{h_{k} h_{k}}$. This remains true even in the case of multiplets of fields of equal conformal weight, as long as their two-point functions can be diagonalized. If this latter situation does no longer hold, which precisely constitutes the logarithmic CFT case [27, then things get more complicated. Now, when computing an OPE $\Psi_{\left(h_{i} ; k_{i}\right)} \Psi_{\left(h_{j} ; k_{j}\right)}$, one needs for each field from a Jordan block $\left\{\Psi_{\left(h_{l} ; k\right)}\right\}_{k=0, \ldots, r-1}$ occurring on the right hand side complete knowledge of all structure constants $C_{\left(h_{i}, h_{j}, h_{l} ; r-1+s\right)}$ and $D_{\left(h_{l} ; r-1+t\right)}$ for $0 \leq s \leq k_{i}+k_{j}, 0 \leq t \leq \min \left(r-1, k_{i}+k_{j}\right)$. Thus, for each triplet of conformal weights $\left(h_{i}, h_{j}, h_{l}\right)$, one needs in fact $2 r-1$ three-point structure constants plus $r$ twopoint structure constants, i.e. in total $3 r-1$ constants, for a rank $r$ LCFT.

Moreover, we discussed how (para-)fermionic and twist fields can be incorporated into our framework. A LCFT can be viewed as an extended ordinary CFT where the space of states has additional sectors. In particular, we showed that any rank two LCFT can consistently accommodate two additional fermionic sectors, and we expect that rank $r$ LCFTs will allow for $\mathbb{Z}_{r}$ para-fermionic sectors. Furthermore, LCFTs naturally incorporate twist fields which considerably enrich the structure of such theories. These twist fields can be considered as the basic entities from which all other fields can be generated by successive application of operator products. Since the OPE of a twist with its anti-twist produces, among others, a logarithmic field, twist fields are also called pre-logarithmic fields [46]. We expect that a more detailed and careful treatment of twist fields in higher rank LCFTs will shed new light on the still mysterious geometric aspects underlying logarithmic conformal field theories (see [19] for some initial remarks on this).

We also presented an BRST-like algebraic argument on the zero mode content, which 
yields necessary conditions for correlators to be non-zero. This could be used to consider Jordan cells with non-proper primary fields and non-quasi-primary logarithmic partners in more detail. In particular, our argument provides conditions for both, the minimal zero mode content of correlators, as well as the maximal zero mode content of the right hand side of OPEs, thus yielding a starting point for a generalized hierarchical scheme.

To conclude, one should remark that we have not yet discussed the most general case. Within this paper, we assumed that the only non-vanishing one-point function is given by the maximal J-level logarithmic partner of the identity, $\left\langle\Psi_{(h=0 ; r-1)}\right\rangle$. However, this might be too restrictive. In particular, a full discussion of logarithmic CFT should include the case of boundaries with their induced spectrum of boundary operators, which possess nonvanishing one-point functions. It would be interesting to compute boundary-boundary OPEs and boundary-bulk OPEs in the LCFT case along the lines of our approach.

ACKnowledgment: We thank Shahin Rouhani and in particular Matthias Gaberdiel for many valuable discussions and comments.

\section{REFERENCES}

[1] D. Bernard, Z. Maassarani, P. Mathieu, Mod.Phys.Lett. A12 (1997) 535 [hep-th/9612217].

[2] M.J. Bhaseen, Nucl.Phys. B604 (2001) 537 [cond-mat/0011229].

[3] M.J. Bhaseen, J.-S. Caux, I.I. Kogan, A.M. Tsvelik, Disordered Dirac Fermions: the Marriage of Three Different Approaches, [cond-mat/0012240].

[4] M.J. Bhaseen, Random Dirac fermions: The su(N) gauge potential and $\mathbb{Z}_{N}$ twists, [cond-mat/0012420]

[5] A. Bilal, I.I. Kogan, Nucl.Phys. B449 (1995) 569 [hep-th/9503209].

[6] A. Campbell-Smith, N.E. Mavromatos, Phys.Lett. B476 (2000) 149 [hep-th/9908139]; Phys.Lett. B488 (2000) 199 [hep-th/0003262].

[7] A. Cappelli, L.S. Georgiev, I.T. Todorov, Comm.Math.Phys. 205 (1999) 657 [hep-th/9810105].

[8] J. Cardy, Logarithmic Correlations in Quenched Random Magnets and Polymers, [cond-mat/9911024].

[9] J.-S. Caux, I.I. Kogan, A.M. Tsvelik, Nucl.Phys. B466 (1996) 444 [hep-th/9511134].

[10] J.-S. Caux, N. Taniguchi, A.M. Tsvelik, Nucl.Phys. B525 (1998) 671 [cond-mat/9801055].

[11] J.-S. Caux, I.I. Kogan, A. Lewis, A.M. Tsvelik, Nucl.Phys. B489 (1997) 469 [hep-th/9606138].

[12] J.-S. Caux, Phys.Rev.Lett. 81 (1998) 4196, [cond-mat/9804133].

[13] W. Eholzer, L. Feher, A. Honecker Nucl.Phys. B518 (1998) 669 [hep-th/9708160].

[14] J.R. Ellis, N.E. Mavromatos, D.V. Nanopoulos, Int.J.Mod.Phys. A12 (1997) 2639 [hep-th/9605046]; Int.J.Mod.Phys. A13 (1998) 1059 [hep-th/9609238]; Gen.Rel.Grav. 32 (2000) 943 [gr-qc/9810086]; Gen.Rel.Grav. 32 (2000) 1777 [gr-qc/9911055]; Phys.Rev. D62 (2000) 084019 [gr-qc/0006004].

[15] J.R. Ellis, N.E. Mavromatos, E. Winstanley, Phys.Lett. B476 (2000) 165 [hep-th/9909068].

[16] M. Flohr, Int.J.Mod.Phys. A11 (1996) 4147 [hep-th/9509166]; Int.J.Mod.Phys. A12 (1997) 1943 [hep-th/9605151]; Nucl.Phys. B514 (1998) 523 [hep-th/9707090]; Null vectors in logarithmic conformal field theory, [hep-th/0009137]. 
[17] M. Flohr, Mod.Phys.Lett. A11 (1996) 55 [hep-th/9605152].

[18] M. Flohr, Nucl.Phys. B482 (1996) 567 [hep-th/9606130].

[19] M. Flohr, Phys.Lett. B444 (1998) 179 [hep-th/9808169].

[20] M. Flohr, M. Krohn, in preparation.

[21] M.R. Gaberdiel, H.G. Kausch, Nucl.Phys. B477 (1996) 293 [hep-th/9604026]; Phys.Lett. B386 (1996) 131 [hep-th/9606050]; Nucl.Phys. B538 (1999) 631 [hep-th/9807091].

[22] M.R. Gaberdiel, Fusion rules and logarithmic representations of a WZW model at fractional level, [hep-th/0105046].

[23] A.M. Ghezelbash, V. Karimipour, Phys.Lett. B402 (1997) 282 [hep-th/9704082].

[24] A.M. Ghezelbash, M. Khorrami, A. Aghamohammadi, Int.J.Mod.Phys. A14 (1999) 2581 [hep-th/9807034].

[25] G. Giribet, Mod.Phys.Lett. A16 (2001) 821 [hep-th/0105248].

[26] E. Gravanis, N.E. Mavromatos, Impulse action on D-particles in Robertson-Walker space times, higher-order logarithmic conformal algebras and cosmological horizons, [hep-th/0106146].

[27] V. Gurarie, Nucl.Phys. B410 (1993) 535 [hep-th/9303160].

[28] V. Gurarie, M. Flohr, C. Nayak, Nucl.Phys. B498 (1997) 513 [cond-mat/9701212].

[29] V. Gurarie, Nucl.Phys. B546 (1999) 765, [cond-mat/9808063].

[30] V. Gurarie, A. Ludwig, Conformal Algebras of 2D Disordered Systems, [cond-mat/9911392].

[31] K. Ino, Phys.Rev.Lett. 81 (1998) 1078 [cond-mat/980333]; Phys.Rev.Lett. 82 (1999) 4902 [cond-mat/9812053]; Phys.Rev.Lett. 83 (1999) 3526, Erratum ibid. 84 (2000) 201 [cond-mat/9906147]; Phys.Rev.Lett. 86 (2001) 882 [cond-mat/0008228].

[32] Y. Ishimoto, Boundary states in boundary logarithmic CFT, [hep-th/0103064].

[33] E.V. Ivashkevich, J.Phys. A 32 (1999) 1691 [cond-mat/9801183].

[34] H.G. Kausch, Curiosities at $c=-2$, [hep-th/9510149]; Nucl.Phys. B583 (2000) 513 [hep-th/0003029].

[35] K. Kaviani, A.M. Ghezelbash, Phys.Lett. B469 (1999) 81 [hep-th/9902104].

[36] S. Kawai, J.F. Wheater, Phys.Lett. B508 (2001) 203 [hep-th/0103197].

[37] F. Kheirandish, M. Khorrami, Eur.Phys.J. C18 (2001) 795 [hep-th/0007013]; Eur.Phys.J.C20 (2001) 593 [hep-th/0007073].

[38] M. Khorrami, A. Aghamohammadi, M.R. Rahimi Tabar, Phys.Lett. B419 (1998) 179 [hep-th/9711155].

[39] M. Khorrami, A. Aghamohammadi, A.M. Ghezelbash, Phys.Lett. B439 (1998) 283 [hep-th/9803071].

[40] J.Y. Kim, H.W. Lee, Y.S. Myung, Origin of logarithmic corrections in threedimensional anti-de Sitter space, [hep-th/9812016].

[41] V.G. Knizhnik, Comm.Math.Phys. 112 (1987) 567.

[42] I.I. Kogan, N.E. Mavromatos, Phys.Lett. B375 (1996) 111 [hep-th/9512210].

[43] I.I. Kogan, C. Mudry, A.M. Tsvelik, Phys.Rev.Lett. 77 (1996) 707, [cond-mat/9602163].

[44] I.I. Kogan, N.E. Mavromatos, J.F. Wheater, Phys.Lett. B387 (1996) 483 [hep-th/9606102].

[45] I.I. Kogan, A. Lewis, O.A. Soloviev, Int.J.Mod.Phys. A13 (1998) 1345 [hep-th/9703028].

[46] I.I. Kogan, A. Lewis, Nucl.Phys. B509 (1998) 687 [hep-th/9705240]; Phys.Lett. B431 (1998) 77 [hep-th/9802102]. 
[47] I.I. Kogan, Phys.Lett. B458 (1999) 66 [hep-th/9903162].

[48] I.I. Kogan, D. Polyakov, Int.J.Mod.Phys. A16 (2001) 2559 [hep-th/0012128].

[49] I.I. Kogan, A.M. Tsvelik, Mod.Phys.Lett. A15 (2000) 931 [hep-th/9912143].

[50] I.I. Kogan, J.F. Wheater, Phys.Lett. B486 (2000) 353 [hep-th/0003184].

[51] I.I. Kogan, A. Nichols, $S U(2)_{0}$ and $O S p(2 \mid 2)_{-2}$ WZNW models: Two current algebras, one Logarithmic CFT, [hep-th/0107160].

[52] G.K. Leontaris, N.E. Mavromatos, Phys.Rev. D61 (2000) 124004 [hep-th/9912230]; Phys.Rev. D64 (2001) 024008 [hep-th/0011102].

[53] A. Lewis, Phys.Lett. B480 (2000) 348 [hep-th/9911163].

[54] A. Lewis, Logarithmic CFT on the boundary and the world-sheet, [hep-th/0009096].

[55] A.W.W. Ludwig, A Free Field Representation of the OSP(2|2) Current Algebra at Level $k=-2$, and Dirac Fermions in a Random SU(2) Gauge Potential, [cond-mat/0012189].

[56] Z. Maassarani, D. Serban, Nucl.Phys. B489 (1997) 603 [hep-th/9605062].

[57] S. Mahieu and P. Ruelle, Scaling fields in the two-dimensional abelian sandpile model, [hep-th/0107150].

[58] N.E. Mavromatos, R.J. Szabo, Phys.Lett. B430 (1998) 94 [hep-th/9803092].

[59] N.E. Mavromatos, R.J. Szabo, Phys.Rev. D59 (1999) 104018 [hep-th/9808124]; Dbrane dynamics and logarithmic superconformal algebras, [hep-th/0106259].

[60] S. Moghimi-Araghi, S. Rouhani, Lett.Math.Phys. 53 (2000) 49 [hep-th/0002142].

[61] S. Moghimi-Araghi, S. Rouhani, M. Saadat, Nucl.Phys. B599 (2001) 531 [hep-th/0008165]; Current algebra associated with logarithmic conformal field theories, [hep-th/0012149].

[62] S. Moghimi-Araghi, S. Rouhani, M. Saadat, On the AdS/CFT correspondence and logarithmic operator, [hep-th/0105123].

[63] Y.S. Myung, H.W. Lee, J.High Energy Phys. 9910 (1999) 009 [hep-th/9904056].

[64] A. Nichols, S. Sanjay, Nucl.Phys. B597 (2001) 633 [hep-th/0007007].

[65] A. Nichols, Logarithmic currents in the $S U(2)_{0}$ WZNW model, [hep-th/0102156].

[66] M.R. Rahimi-Tabar, S. Rouhani, Europhys.Lett. C37 (1997) 447 [hep-th/9606143]; Logarithmic Correlation Functions in Two Dimensional Turbulence, [hep-th/9606154].

[67] M.R. Rahimi-Tabar, A. Aghamohammadi, M. Khorrami, Nucl.Phys. B497 (1997) 555 [hep-th/9610168].

[68] M.R. Rahimi-Tabar, S. Rouhani, Phys.Lett. B431 (1998) 85 [hep-th/9707060].

[69] M. Reza Rahimi-Tabar, Nucl.Phys. B588 (2000) 630 [cond-mat/0002309].

[70] N. Read, D. Green, Paired States of Fermions in Two-Dimensions with Breaking of Parity and Time Reversal Symmetries, and the Fractional Quantum Hall Effect, [cond-mat/9906453].

[71] N. Read, H. Saleur, Exact spectra of conformal supersymmetric nonlinear sigma models in two dimensions, [hep-th/0106124].

[72] F. Rohsiepe, On reducible but indecomposable representations of the Virasoro algebra, [hep-th/9611160].

[73] L. Rozansky, H. Saleur, Nucl.Phys. B376 (1992) 461; Nucl.Phys. B389 (1993) 365, [hep-th/9203069].

[74] H. Saleur, Nucl.Phys. B489 (1992) 486, [hep-th/9111007].

[75] S. Sanjay, Logarithmic operators in $S L(2, R)$ WZNW model, singletons and $A d S_{3} /(L) C F T_{2}$ correspondence, [hep-th/0011056].

[76] S. Skoulakis, S. Thomas, Phys.Lett. B438 (1998) 301 [cond-mat/9802040]. 